\title{
单位球面 $\boldsymbol{S}^{6}$ 中的 Blaschke 等参超曲面
}

\author{
李兴校*，彭业娟 \\ 河南师范大学数学与信息科学学院, 新乡 453007 \\ E-mail: xxl@henannu.edu.cn, yejuan666@126.com
}

收稿日期: 2009-07-24; 接受日期: 2010-07-08; * 通信作者

国家自然科学基金 (批准号: 10671181)、河南省基础与前沿技术计划 (批准号: 092300410143) 和河南省教育厅自然科学研究计划 (批 准号: 2009A110010) 资助项目

摘要 单位球面中的一个无脐点浸入子流形称为 Blaschke 等参子流形如果它的 Möbius 形式恒为零 并且所有的 Blaschke 特征值均为常数. 维数 $m \leqslant 4$ 的 Blaschke 等参超曲面已经有了完全的分类. 截止目前, Möbius 等参超曲面的所有已知例子都是 Blaschke 等参的. 另一方面, 确实存在许多不是 Möbius 等参的 Blaschke 等参超曲面, 它们都具有不超过两个的不同 Blaschke 特征值. 在已有分类定 理的基础上, 本文对于 5 维 Blaschke 等参超曲面进行了完全的分类. 特别地, 我们证明了 $S^{6}$ 中具有 多于两个不同 Blaschke 特征值的 Blaschke 等参超曲面一定是 Möbius 等参的, 给出了此前一个问题 的部分解答.

关键词 Blaschke 等参超曲面 Möbius 形式 Blaschke 张量 Möbius 度量 Möbius 第二基本形式

MSC (2000) 主题分类 $53 \mathrm{~A} 30,53 \mathrm{~B} 25$

\section{1 引言}

设 $x: M^{m} \rightarrow S^{n}(n=m+p)$ 是单位球面 $S^{n}$ 中的一个无脐点浸入子流形. 文献 [1] 中引入了 $x$ 的 四个基本 Möbius 不变量: Möbius 度量 $g 、$ Möbius 形式 $\Phi 、$ Blaschke 张量 $A$ 和 Möbius 第二基本形式 $B$. 作者同时建立了 $S^{n}$ 中无脐点子流形的 Möbius 基本定理 (存在唯一性定理), 其基础是一组由上述 四个 Möbius 不变量完全确定的 Möbius 运动方程. 因此, 对这些不变量的研究在子流形的 Möbius 几 何中是至关重要的. 近年来, 相关的研究有了长足的进展, 得到了许多有意义的结果, 特别是得到了一 些重要的分类定理. 比如: 对于 $S^{n}$ 中的 Möbius 迷向子流形的分类 ${ }^{[2]}$; 对于空间形式中具有常平均曲 率 (平行平均曲率向量) 和常 Möbius 数量曲率的超曲面 (子流形) 的 Möbius 刻画 ${ }^{[3,4]}$; 对于 $S^{m+1}$ 中 具有平行 Möbius 第二基本形式 ${ }^{[5]}$ 或具有平行 Blaschke 张量的超曲面 ${ }^{[6]}$ 的分类; 等等. 作为这些结 果的进一步推广, 具有平行的所谓仿 Blaschke 张量 ( $D^{\lambda}:=A+\lambda B, \lambda$ 是一个给定的常数) 的超曲面在 文献 [7] 中得到了完全的分类; 同时, 文献 [8] 则讨论了具有零 Möbius 形式、并且其仿 Blaschke 张量 $D^{\lambda}$ 恰有两个不同常数特征值的超曲面, 也得到了一个分类定理.

如所知, 对于超曲面, 其 Möbius 第二基本形式 $B$ 的特征值称为它的Möbius 主曲率. 而对于子 流形, 其 Blaschke 张量 $A$ 的特征值可自然地称为它的Blaschke 特征值. 下述 Möbius 等参超曲面和 Blaschke 等参子流形的定义分别来自于文献 [9] 和 [10]:

定理 1.1 设 $x: M^{m} \rightarrow S^{m+p}$ 是单位球面 $S^{n}$ 中的无脐点浸入子流形, 其 Möbius 形式 $\Phi \equiv 0$. 
(1) 如果 $p=1$, 并且 $x$ 的所有 Möbius 主曲率均为常数, 则称 $x$ 为单位球面 $S^{m+1}$ 中的 Möbius 等 参超曲面 ${ }^{[9]}$;

(2) 如果 $x$ 的所有 Blaschke 特征值均为常数, 则称 $x$ 为单位球面 $S^{m+p}$ 中的 Blaschke 等参子流 形 $[10,11]$.

需要说明的是, 定义 1.1 中的条件 $\Phi \equiv 0$ 在子流形的 Möbius 几何中是一个简单、自然而重要的 条件. 在此条件下, 已有一系列非常有价值的结果. 相关的细节可参阅文献 [2-4, 12-15].

如所知, Möbius 等参超曲面和所谓的 Dupin 超曲面有密切的关系. 比如: Möbius 等参超曲面都是 proper Dupin 超曲面, 而具有两个不同主曲率的 Dupin 超曲面一定是 Möbius 等参的 [9].

近年来, Möbius 等参超曲面得到了广泛深入的研究. 特别地, Möbius 等参超曲面的一些子类得到 了完全的分类. 比如: 具有两个不同 Möbius 主曲率或维数 $m \leqslant 5$ 的 Möbius 等参超曲面已经被完全 分类, 其细节可参见文献 $[9,16-19]$.

和 Möbius 等参超曲面一样, Blaschke 等参超曲面的分类问题是自然的, 也是有意义的. 与此同时, Möbius 等参超曲面和 Blaschke 等参超曲面之间的密切联系也是子流形的 Möbius 几何中令人感兴趣 的问题. 单从可积条件就可看出这两类超曲面具有很多类似的性质. 然而, 这种类似究竟能达到什么 样的程度则有待更深入的研讨. 注意到, 一方面, Möbius 等参超曲面所有已知的例子都是 Blaschke 等 参的; 另一方面也确实存在许多不是 Möbius 等参的 Blaschke 等参超曲面, 它们都具有不超过两个的 Blaschke 特征值. 基于上述事实, 文献 [10] 提出了如下的问题:

问题 [10] 设 $x: M^{m} \rightarrow S^{m+1}$ 是 Blaschke 等参超曲面, 具有超过两个的不同 Blaschke 特征值. 那么, $x$ 一定是 Möbius 等参超曲面吗?

截止目前, 上述问题已经有了部分肯定的解答, 也没有发现任何反例 (参见文献 $[10,20]$ ). 特别地, 我们有

定理 $1.1^{[10,11,20]}$ 设 $x: M^{m} \rightarrow S^{m+1}$ 是一个 Blaschke 等参超曲面, 具有超过两个的不同 Blaschke 特征值. 如果 (1) $m=3,4$; 或 (2) $m=5,6$ 并且 $x$ 的每一个 Blaschke 特征值都是单的; 或 (3) $x$ 具有三个不同的 Blaschke 特征值, 并且其中有一个是单的, 则 $x$ 一定是 Möbius 等参的.

有关 Blaschke 等参子流形尤其是 Blaschke 等参超曲面的分类问题, 一些有意义的特殊情形先后 在文献 $[2,11,10,20]$ 中被考虑. 特别地, Möbius 迷向子流形 (即只有一个不同 Blaschke 特征值的 Blaschke 等参子流形) 在文献 [2] 中得到了完全的分类; 文献 [11] 中则对具有两个不同 Blaschke 特征 值的 Blaschke 等参超曲面和 3 维 Blaschke 等参超曲面进行了分类; 4 维 Blaschke 等参超曲面在文献 [10] 中有了分类定理. 最近, 具有三个不同 Blaschke 特征值且其中有一个为单的 Blaschke 等参超曲面 也被完全分类 (见文献 [20]). 为了本文应用的需要和方便读者, 上述的分类结果归纳如下:

定理 1.2 ${ }^{[2,11]}$ 设 $x: M^{m} \rightarrow S^{m+1}(m \geqslant 2)$ 是具有不超过两个不同 Blaschke 特征值的 Blaschke 等参超曲面. 则 $x$ 局部地 Möbius 等价于下列超曲面之一:

(1) $S^{m+1}$ 中具有常数量曲率的极小浸入超曲面;

(2) $\mathbb{R}^{m+1}$ 中具有常数量曲率的极小浸入超曲面在共形映射 $\sigma: \mathbb{R}^{m+1} \rightarrow S^{m+1} \backslash\{(-1,0)\}$ 下的像;

(3) $H^{m+1}$ 中具有常数量曲率的极小浸入超曲面在共形映射 $\tau: H^{m+1} \rightarrow S_{+}^{m+1} \subset S^{m+1}$ 下的像;

(4) $S^{m+1}$ 中的标准环面 $S^{K}(r) \times S^{m-K}\left(\sqrt{1-r^{2}}\right)$, 这里的实数 $r \in(0,1)$, 正整数 $K \leqslant m-1$;

(5) $\mathbb{R}^{m+1}$ 中的标准柱面 $S^{K}(r) \times \mathbb{R}^{m-K}$ 在 $\sigma$ 下的像, 其中 $r>0, K \leqslant m-1$;

(6) $H^{m+1}$ 中的标准柱面 $S^{K}(r) \times H^{m-K}\left(-\frac{1}{1+r^{2}}\right)$ 在 $\tau$ 下的像, 其中 $r>0, K \leqslant m-1$;

(7) 例 3.2 中满足 $\lambda=0$ 的极小超曲面;

(8) 例 3.3 中满足 $\lambda=0$ 的非极小超曲面. 
本定理中及此后所提到的共形映射 $\sigma$ 和 $\tau$ 的定义由第 2 节中的 (2.1) 和 (2.2) 给出.

定理 $1.3^{[20]}$ 设 $x: M^{m} \rightarrow S^{m+1}$ 是 Blaschke 等参超曲面, 具有三个不同的 Blaschke 特征值并 且其中有一个是单的. 则 $x$ 局部上 Möbius 等价于

(1) 例 3.1 中由某一组参数 $p, q, r$ 确定的超曲面 $\operatorname{CSS}(p, q, r)$, 或者

(2) $S^{4}$ 中具有三个不同主曲率的非极小 Cartan 等参超曲面 (即 $S^{2}(\sqrt{3})$ 到 $S^{4}$ 中的标准 Veronese 浸入的管状曲面), 或者

(3) 例 3.2 中的一个超曲面, 其中 $K=3, \tilde{y}_{1}: M_{1} \rightarrow S^{4}(r)$ 是具有三个不同主曲率 $\mu_{1}, \mu_{2}, \mu_{3}$ 的非 极小 Cartan 等参超曲面, 并且存在某个 $i \in\{1,2,3\}$ 使得 $\lambda \mu_{i}=\frac{1}{r^{2}}$.

显然, 下一步要考虑的是维数 $m \geqslant 5$ 的 Blaschke 等参超曲面的分类问题. 本文对 5 维 Blaschke 等参超曲面进行了完全的分类. 主要定理如下:

定理 1.4 设 $x: M^{5} \rightarrow S^{6}$ 是 Blaschke 等参超曲面. 则 $x$ 局部地 Möbius 等价于下列超曲面中 的一个:

(1) $S^{6}$ 中具有常数量曲率的极小浸入超曲面;

(2) $\mathbb{R}^{6}$ 中具有常数量曲率的极小浸入超曲面在共形映射 $\sigma$ 下的像;

(3) $H^{6}$ 中具有常数量曲率的极小浸入超曲面在共形映射 $\tau$ 下的像;

(4) $S^{6}$ 中的标准环面 $S^{K}(r) \times S^{5-K}\left(\sqrt{1-r^{2}}\right)$, 其中 $r \in(0,1), K=1,2,3,4$;

(5) $\mathbb{R}^{6}$ 中的标准柱面 $S^{K}(r) \times \mathbb{R}^{5-K}$ 在共形映射 $\sigma$ 下的像, 其中 $r>0, K=1,2,3,4$;

(6) $H^{6}$ 中的标准柱面 $S^{K}(r) \times H^{5-K}\left(-\frac{1}{1+r^{2}}\right)$ 在共形映射 $\tau$ 下的像, 其中 $r>0, K=1,2,3,4$;

(7) 例 3.2 中满足 $\lambda=0$ 的极小超曲面, 其中 $m=5, K=2,3,4$;

(8) 例 3.3 中满足 $\lambda=0$ 的非极小超曲面, 其中 $m=5, K=2,3,4$;

(9) 例 3.1 中的超曲面 $\operatorname{CSS}(p, q, r)$, 其中 $m=5$;

(10) 例 3.2 中的超曲面, 其中 $m=5, K=3, r>0$, 并且 $\tilde{y}_{1}$ 是 $S^{4}(r)$ 中具有三个不同主曲率的 Cartan 等参超曲面;

(11) 例 3.2 中的超曲面, 其中 $m=5, K=4, r>0$, 并且 $\tilde{y}_{1}$ 是 $S^{5}(r)$ 中具有四个不同主曲率的 Euclid 等参超曲面.

注记 1.1 在定理 1.4 的证明过程中, 关键是证明了 $S^{6}$ 中具有四个不同 Blaschke 特征值的 Blaschke 等参超曲面一定是 Möbius 等参的 (参见命题 4.2). 结合定理 1.1, 可得如下的定理:

定理 1.56 维单位球面 $S^{6}$ 中具有多于两个不同 Blaschke 特征值的 Blaschke 等参超曲面必为 Möbius 等参超曲面.

\section{2 预备知识}

设 $\mathbb{R}^{n}$ 是 $n$ 维 Euclid 空间, 其标准内积用点 “.” 表示, $\mathbb{R}_{1}^{N} \equiv \mathbb{R}_{1} \times \mathbb{R}^{N-1}$ 是 $N$ 维 Lorentz 空间, 相 应的标准 Lorentz 内积 $\langle\cdot, \cdot\rangle_{1}$ 的定义是

$$
\left\langle y, y^{\prime}\right\rangle_{1}=-y_{0} y_{0}^{\prime}+y_{1} \cdot y_{1}^{\prime}, \quad y=\left(y_{0}, y_{1}\right), \quad y^{\prime}=\left(y_{0}^{\prime}, y_{1}^{\prime}\right) \in \mathbb{R}_{1}^{N} .
$$

再设 $S^{n}(r)$ 是半径为 $r$ 的 $n$ 维标准球面, $H^{n}(c)$ 是具常曲率 $c<0$ 的 $n$ 维双曲空间, 其定义如下:

$$
H^{n}(c)=\left\{y=\left(y_{0}, y_{1}\right) \in \mathbb{R}_{1}^{n+1} ;\langle y, y\rangle_{1}=1 / c, y_{0}>0\right\} .
$$

以下记 $S^{n}=S^{n}(1), H^{n}=H^{n}(-1)$. 
用 $S_{+}^{n}$ 表示单位球面 $S^{n}$ 中第一个坐标为正数的半球面, 则有两个典型的共形微分同胚 $\sigma: \mathbb{R}^{n} \rightarrow$ $S^{n} \backslash\{(-1,0)\}, \tau: H^{n} \rightarrow S_{+}^{n}$, 分别定义如下:

$$
\begin{aligned}
\sigma(u) & =\left(\frac{1-|u|^{2}}{1+|u|^{2}}, \frac{2 u}{1+|u|^{2}}\right), \quad u \in \mathbb{R}^{n}, \\
\tau(y) & =\left(\frac{1}{y_{0}}, \frac{y_{1}}{y_{0}}\right), \quad y=\left(y_{0}, y_{1}\right) \in H^{n} \subset \mathbb{R}_{1}^{n+1} .
\end{aligned}
$$

设 $x: M^{m} \rightarrow S^{n}$ 是无脐点的浸入子流形, $h$ 是 $x$ 的第二基本形式, $H$ 是 $x$ 的平均曲率向量场. 令

$$
\rho=\left(\frac{m}{m-1}\left(|h|^{2}-m|H|^{2}\right)\right)^{\frac{1}{2}}, \quad Y=\rho(1, x) .
$$

则 $Y: M^{m} \rightarrow \mathbb{R}_{1}^{n+2}$ 是 $M^{m}$ 到 Lorentz 空间 $\mathbb{R}_{1}^{n+2}$ 的浸入, 称为 $x$ 的典型提升 (或Möbius 位置向量); 由 (2.3) 确定的函数 $\rho$ 称为 $x$ 的Möbius 因子. 令

$$
C_{+}^{n+1}=\left\{Y=\left(Y_{0}, Y\right) \in \mathbb{R}_{1} \times \mathbb{R}^{n+1} ;\langle Y, Y\rangle_{1}=0, Y_{0}>0\right\} .
$$

设 $O(n+1,1)$ 是 $\mathbb{R}_{1}^{n+2}$ 上的所有保持标准 Lorentz 内积 $\langle\cdot, \cdot\rangle_{1}$ 不变的线性变换构成的 Lorentz 正 交群. $O(n+1,1)$ 有一个子群 $O^{+}(n+1,1)$, 其定义为

$$
O^{+}(n+1,1)=\left\{T \in O(n+1,1) ; T\left(C_{+}^{n+1}\right) \subset C_{+}^{n+1}\right\} .
$$

定理 $2.1^{[1]}$ 分别以 $Y$ 和 $\tilde{Y}$ 为 Möbius 位置向量的两个子流形 $x, \tilde{x}: M^{m} \rightarrow S^{n}$ 是 Möbius 等价 的当且仅当存在 $T \in O^{+}(n+1,1)$ 使得 $\tilde{Y}=T(Y)$.

根据定理 2.1, Lorentz 内积 $\langle\cdot, \cdot\rangle_{1}$ 在 $M^{m}$ 上的诱导度量 $g=Y^{*}\langle\cdot, \cdot\rangle_{1}=\rho^{2} d x \cdot d x$ 是一个 Möbius 不 变的 Rieman 度量 (参见文献 $[1,21]$ ), 称为 $x$ 的Möbius 度量. 利用向量值函数 $Y$ 和度量 $g$ 的 Laplace 算子 $\Delta$, 可以定义另外一个重要的向量函数 $N: M^{m} \rightarrow \mathbb{R}_{1}^{n+2}$ :

$$
N=-\frac{1}{m} \Delta Y-\frac{1}{2 m^{2}}\langle\Delta Y, \Delta Y\rangle_{1} Y .
$$

则有 $\langle N, N\rangle_{1}=0,\langle N, Y\rangle_{1}=1$.

设 $V \rightarrow M^{m}$ 是向量从 $\mathbb{R} Y \oplus \mathbb{R} N \oplus Y_{*}\left(T M^{m}\right)$ 关于 Lorentz 内积 $\langle\cdot, \cdot\rangle_{1}$ 在 Lorentz 平凡丛 $M^{m} \times \mathbb{R}_{1}^{n+2}$ 中的正交补, 它称为浸入 $x$ 的Möbius 法丛.

为方便起见, 本节中的指标取值范围如下:

$$
1 \leqslant i, j, k \leqslant m, \quad m+1 \leqslant \alpha, \beta, \gamma \leqslant n .
$$

对于诱导度量 $d x \cdot d x$ 的任意单位正交标架场 $\left\{e_{i}\right\}$, 其对偶记为 $\left\{\theta^{i}\right\}$. 用 $\left\{e_{\alpha}\right\}$ 表示 $x$ 的单位正交 法标架场, 令

$$
E_{i}=\rho^{-1} e_{i}, \quad \omega^{i}=\rho \theta^{i}, \quad E_{\alpha}=f\left(e_{\alpha}\right),
$$

则 $\left\{E_{i}\right\}$ 是 $M$ 上关于 Möbius 度量 $g$ 的局部单位正交标架场, $\left\{\omega^{i}\right\}$ 是 $\left\{E_{i}\right\}$ 的对偶, $\left\{E_{\alpha}\right\}$ 是 Möbius 法 丛 $V$ 的局部单位正交标架场. 由文献 $[1,2]$, Möbius 不变量 $\Phi, A$ 和 $B$ 具有如下的局部表达式:

$$
\Phi=\sum C_{i}^{\alpha} \omega^{i} E_{\alpha}, \quad A=\sum A_{i j} \omega^{i} \omega^{j}, \quad B=\sum B_{i j}^{\alpha} \omega^{i} \omega^{j} E_{\alpha}
$$


其中

$$
\begin{aligned}
C_{i}^{\alpha}= & -\rho^{-2}\left(H_{, i}^{\alpha}+\sum\left(h_{i j}^{\alpha}-H^{\alpha} \delta_{i j}\right) e_{j}(\log \rho)\right), \\
A_{i j}= & -\rho^{-2}\left((\log \rho)_{, i j}-e_{i}(\log \rho) e_{j}(\log \rho)-\sum H^{\alpha} h_{i j}^{\alpha}\right) \\
& -\frac{1}{2} \rho^{-2}\left(|d \log \rho|^{2}-1+|H|^{2}\right) \delta_{i j}, \\
B_{i j}^{\alpha}= & \rho^{-1}\left(h_{i j}^{\alpha}-H^{\alpha} \delta_{i j}\right) .
\end{aligned}
$$

这里下标 “, $i$ ” 表示关于诱导度量 $d x \cdot d x$ 的沿方向 $e_{i}$ 的协变导数.

用 $R_{i j k l}, R_{i j}$ 分别表示 Möbius 度量 $g$ 的 Riemann 曲率张量和 Ricci 张量在标架场 $\left\{E_{i}\right\}$ 下的分 量, $\kappa$ 表示法化的 Möbius 数量曲率, 则下述恒等式成立 ${ }^{[1]}$ :

$$
\begin{aligned}
& \operatorname{tr} A=\frac{1}{2 m}\left(1+m^{2} \kappa\right), \quad \operatorname{tr} B=\sum_{i, \alpha} B_{i i}^{\alpha} E_{\alpha}=0, \quad|B|^{2}=\sum_{i, j, \alpha}\left(B_{i j}^{\alpha}\right)^{2}=\frac{m-1}{m}, \\
& R_{i j k l}=\sum\left(B_{i l}^{\alpha} B_{j k}^{\alpha}-B_{i k}^{\alpha} B_{j l}^{\alpha}\right)+A_{i l} \delta_{j k}-A_{i k} \delta_{j l}+A_{j k} \delta_{i l}-A_{j l} \delta_{i k} .
\end{aligned}
$$

如果 $C_{i j}^{\alpha}, A_{i j k}, B_{i j k}^{\alpha}$ 分别是 $\Phi, A, B$ 的协变导数在标架场 $\left\{E_{i}\right\}$ 和 $\left\{E_{\alpha}\right\}$ 下的分量, 则有 ${ }^{[1]}$ :

$$
\begin{aligned}
& C_{i j}^{\alpha}-C_{j i}^{\alpha}=\sum\left(B_{i k}^{\alpha} A_{k j}-B_{k j}^{\alpha} A_{k i}\right), \\
& A_{i j k}-A_{i k j}=\sum\left(B_{i k}^{\alpha} C_{j}^{\alpha}-B_{i j}^{\alpha} C_{k}^{\alpha}\right), \\
& B_{i j k}^{\alpha}-B_{i k j}^{\alpha}=\delta_{i j} C_{k}^{\alpha}-\delta_{i k} C_{j}^{\alpha} .
\end{aligned}
$$

特别地, 如果 Möbius 形式 $\Phi \equiv 0$, 则 Blaschke 张量 $A$ 和 Möbius 第二基本形式 $B$ 都是 Codazzi 张量.

求 $(2.13)$ 和 $(2.16)$ 式的迹可得

$$
\begin{aligned}
& R_{i j}=-\sum B_{i k}^{\alpha} B_{k j}^{\alpha}+\operatorname{tr} A \delta_{i j}+(m-2) A_{i j}, \\
& (m-1) C_{i}^{\alpha}=-\sum B_{i j j}^{\alpha} .
\end{aligned}
$$

若 $m \geqslant 3$ 并且 $x$ 是超曲面, 则根据 (2.12), (2.17) 和 (2.18), Möbius 形式 $\Phi$ 和 Blaschke 张量 $A$ 可由 Möbius 度量 $g$ 和 Möbius 第二基本形式 $B$ 完全确定. 因此有下述定理:

定理 $2.2^{[1]}$ 设 $m \geqslant 3$. 则两个超曲面 $x: M^{m} \rightarrow S^{m+1}, \tilde{x}: \tilde{M}^{m} \rightarrow S^{m+1}$ Möbius 等价当且仅当 存在保持 Möbius 度量和 Möbius 第二基本形式不变的微分同胚 $\varphi: M^{m} \rightarrow \tilde{M}^{m}$.

\section{Blaschke 等参超曲面的例子}

为简化主要定理的证明和方便读者, 本节将列出引言中提到的部分典型 Blaschke 等参超曲面. 有 关细节可以在相应的参考文献中找到.

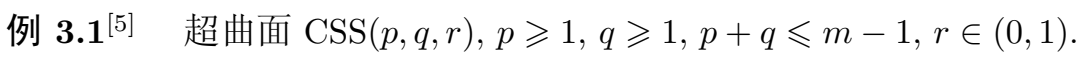

设 $\mathbb{R}^{+}$是所有正数之集. 对于任意两个满足 $p+q \leqslant m-1$ 的自然数 $p, q$ 和正数 $r \in(0,1)$, 考虑 浸入超曲面

$$
u: S^{p}(r) \times S^{q}\left(\sqrt{1-r^{2}}\right) \times \mathbb{R}^{+} \times \mathbb{R}^{m-p-q-1} \rightarrow \mathbb{R}^{m+1}, \quad u=\left(t u^{\prime}, t u^{\prime \prime}, u^{\prime \prime \prime}\right),
$$


其中

$$
u^{\prime} \in S^{p}(r) \subset \mathbb{R}^{p+1}, \quad u^{\prime \prime} \in S^{q}\left(\sqrt{1-r^{2}}\right) \subset \mathbb{R}^{q+1}, \quad t \in \mathbb{R}^{+}, \quad u^{\prime \prime \prime} \in \mathbb{R}^{m-p-q-1} .
$$

则 $x=\sigma \circ u$ 是 $S^{m+1}$ 中的一个无脐点浸入超曲面. 这个超曲面在文献 [5] 中被表示为 $\operatorname{CSS}(p, q, r)$. 直接 计算可知, $\operatorname{CSS}(p, q, r)$ 的 Blaschke 张量 $A$ 和 Möbius 第二基本形式 $B$ 都是平行的, 因而其 Möbius 形 式恒为零 (参见文献 $[5,6]$ ). 此外, $\operatorname{CSS}(p, q, r)$ 恰好具有三个不同的 Möbius 主曲率, 其 Blaschke 特征 值则由下式给出

$$
\begin{aligned}
& A_{1}=\cdots=A_{p}=-\frac{m-1}{2 m^{2} Q}\left((m-p-q)^{2} r^{4}+\left(2 m(2 p+q)-2 p(p+q)-m^{2}\right) r^{2}-p(2 m-p)\right), \\
& A_{p+1}=\cdots=A_{p+q+1}=\frac{m-1}{2 m^{2} Q}\left(m^{2} r^{2}-\left(p+(m-p-q) r^{2}\right)^{2}\right), \\
& A_{p+q+1}=\cdots=A_{m}=\frac{m-1}{2 m^{2} Q}\left(\left(m^{2}-(p+q)^{2}\right) r^{4}+\left(2 p(p+q)-m^{2}\right) r^{2}-p^{2}\right),
\end{aligned}
$$

其中

$$
Q=q(m-q) r^{4}+2 p q r^{2}\left(1-r^{2}\right)+p(m-p)\left(1-r^{2}\right)^{2},
$$

注记 3.1 不难知道, 上述超曲面 $\operatorname{CSS}(p, q, r)$ 在一般情况下有三个不同的 Blaschke 特征值. 但 在 $r^{2}=\frac{p}{p+q}$ 时, $\operatorname{CSS}(p, q, r)$ 只有两个不同的 Blaschke 特征值.

例 3.2 ${ }^{[6-8,11]}$ 设 $\lambda \in \mathbb{R}$. 对满足 $m \geqslant 3$ 和 $2 \leqslant K \leqslant m-1$ 的整数 $m, K$, 设 $\tilde{y}_{1}: M_{1} \rightarrow S^{K+1}(r) \subset$ $\mathbb{R}^{K+2}$ 是一个无脐点的浸入超曲面, 其数量曲率 $S_{1}$ 和平均曲率 $H_{1}$ 满足

$$
S_{1}=\frac{m K(K-1)-(m-1) r^{2}}{m r^{2}}+m(m-1) \lambda^{2}, \quad H_{1}=-\frac{m}{K} \lambda .
$$

再设

$$
\tilde{y}=\left(\tilde{y}_{0}, \tilde{y}_{2}\right): H^{m-K}\left(-\frac{1}{r^{2}}\right) \rightarrow \mathbb{R}_{1}^{m-K+1}
$$

是标准嵌入. 定义

$$
\tilde{M}^{m}=M_{1} \times H^{m-K}\left(-\frac{1}{r^{2}}\right), \quad \tilde{Y}=\left(\tilde{y}_{0}, \tilde{y}_{1}, \tilde{y}_{2}\right) .
$$

则 $\tilde{Y}: \tilde{M}^{m} \rightarrow \mathbb{R}_{1}^{m+3}$ 是满足条件 $\langle\tilde{Y}, \tilde{Y}\rangle_{1}=0$ 的浸入, 它在 $\tilde{M}^{m}$ 上诱导了一个 Riemann 度量

$$
g=\langle d \tilde{Y}, d \tilde{Y}\rangle_{1}=-d \tilde{y}_{0}^{2}+d \tilde{y}_{1}^{2}+d \tilde{y}_{2}^{2} .
$$

显然, 作为 Riemann 流形有

$$
\left(\tilde{M}^{m}, g\right)=\left(M_{1}, d \tilde{y}_{1}^{2}\right) \times\left(H^{m-K}\left(-\frac{1}{r^{2}}\right),\langle d \tilde{y}, d \tilde{y}\rangle_{1}\right) .
$$

定义

$$
\tilde{x}_{1}=\frac{\tilde{y}_{1}}{\tilde{y}_{0}}, \quad \tilde{x}_{2}=\frac{\tilde{y}_{2}}{\tilde{y}_{0}}, \quad \tilde{x}=\left(\tilde{x}_{1}, \tilde{x}_{2}\right) .
$$

则 $|\tilde{x}|^{2}=1$ 并且 $\tilde{x}: \tilde{M}^{m} \rightarrow S^{m+1}$ 是无脐点的浸入超曲面, 以 $\tilde{Y}$ 为其 Möbius 位置向量.

设 $\left\{E_{i}, 1 \leqslant i \leqslant K\right\},\left\{E_{i}, K+1 \leqslant i \leqslant m\right\}$ 分别是 $\left(M_{1}, d \tilde{y}^{2}\right)$ 和 $H^{m-K}\left(-\frac{1}{r^{2}}\right)$ 上的单位正交标架场. 则 $\left\{E_{i}, 1 \leqslant i \leqslant m\right\}$ 是 $\left(\tilde{M}^{m}, g\right)$ 上的单位正交标架场, 其对偶标架场记为 $\left\{\omega^{i}, 1 \leqslant i \leqslant m\right\}$. 等距浸入 
$\tilde{y}_{1}$ 的第二基本形式记为 $h=\sum_{i, j=1}^{K} h_{i j} \omega^{i} \omega^{j}$. 经直接计算可得 (参见文献 [7] 或 [8]), $\tilde{x}$ 的 Blaschke 张 量 $A=\sum A_{i j} \omega^{i} \omega^{j}$ 和 Möbius 第二基本形式 $B=\sum B_{i j} \omega^{i} \omega^{j}$ 由下列式子给出:

$$
\begin{aligned}
& A_{i j}=\left(\frac{1}{2 r^{2}}-\frac{1}{2} \lambda^{2}\right) \delta_{i j}-\lambda h_{i j}, \quad B_{i j}=h_{i j}+\lambda \delta_{i j}, \quad \text { 如果 } 1 \leqslant i, j \leqslant K ; \\
& A_{i j}=-\left(\frac{1}{2 r^{2}}+\frac{1}{2} \lambda^{2}\right) \delta_{i j}, \quad B_{i j}=\lambda \delta_{i j}, \quad \text { 如果 } K+1 \leqslant i, j \leqslant m \text {; } \\
& A_{i j}=0, \quad B_{i j}=0, \quad \text { 其他. }
\end{aligned}
$$

因此, $\tilde{x}$ 有常数 Blaschke 特征值当且仅当浸入 $\tilde{y}_{1}: M_{1} \rightarrow S^{K+1}(r)$ 是 (Euclid) 等参超曲面. 在此情况 下, $\tilde{x}$ 的 Möbius 形式恒为零, 因而是 Blaschke 等参超曲面.

注记 3.2 例 3.2 中的超曲面 $\tilde{x}$ 是 Blaschke 等参并且具有三个不同 Blaschke 特征值和四个不同 Möbius 主曲率当且仅当相应的 $\tilde{y}_{1}$ 是具有三个不同主曲率 $\mu_{1}, \mu_{2}, \mu_{3}$ 的非极小 (Euclid) 等参超曲面, 并且对某个 $i \in\{1,2,3\}$ 满足 $\lambda \mu_{i}=\frac{1}{r^{2}}$. 根据 Cartan 在文献 [22] 中的讨论 (也可参阅文献 [23]), 这样 的超曲面 $\tilde{y}_{1}$ 的确存在, 并且其所有的主曲率 $\mu_{1}, \mu_{2}, \mu_{3}$ 必须有相同的重数. 特别地, 如果 $\tilde{x}$ 有一个单 的 Blaschke 特征值, 则 $K=\operatorname{dim} M_{1}=3$. 此外, 如果 $\tilde{x}$ 至少有四个不同的 Blaschke 特征值, 则对每一 个 $i \in\{1,2,3\}, \lambda \mu_{i} \neq \frac{1}{r^{2}}$, 或者 $\tilde{y}_{1}$ 是具有不少于四个不同主曲率的等参超曲面.

例 $\mathbf{3 . 3}^{[6-8,11]}$ 设 $\lambda \in \mathbb{R}, m \geqslant 3,2 \leqslant K \leqslant m-1, \tilde{y}=\left(\tilde{y}_{0}, \tilde{y}_{1}\right): M_{1} \rightarrow H^{K+1}\left(-\frac{1}{r^{2}}\right) \subset \mathbb{R}_{1}^{K+2}$ 是一 个无脐点的浸入超曲面, 其数量曲率 $S_{1}$ 和平均曲率 $H_{1}$ 由下式给出

$$
S_{1}=-\frac{m K(K-1)+(m-1) r^{2}}{m r^{2}}+m(m-1) \lambda^{2}, \quad H_{1}=-\frac{m}{K} \lambda .
$$

再设

$$
\tilde{y}_{2}: S^{m-K}(r) \rightarrow \mathbb{R}^{m-K+1}
$$

是标准嵌入. 令

$$
\tilde{M}^{m}=M_{1} \times S^{m-K}(r), \quad \tilde{Y}=\left(\tilde{y}_{0}, \tilde{y}_{1}, \tilde{y}_{2}\right) .
$$

则 $\tilde{Y}: \tilde{M}^{m} \rightarrow \mathbb{R}_{1}^{m+3}$ 是满足 $\langle\tilde{Y}, \tilde{Y}\rangle_{1}=0$ 的浸入, 它在 $\tilde{M}^{m}$ 上诱导了一个 Riemann 度量

$$
g=\langle d \tilde{Y}, d \tilde{Y}\rangle_{1}=-d \tilde{y}_{0}^{2}+d \tilde{y}_{1}^{2}+d \tilde{y}_{2}^{2} .
$$

定义

$$
\tilde{x}_{1}=\frac{\tilde{y}_{1}}{\tilde{y}_{0}}, \quad \tilde{x}_{2}=\frac{\tilde{y}_{2}}{\tilde{y}_{0}}, \quad \tilde{x}=\left(\tilde{x}_{1}, \tilde{x}_{2}\right) .
$$

则 $|\tilde{x}|^{2}=1$ 并且 $\tilde{x}: \tilde{M}^{m} \rightarrow S^{m+1}$ 是无脐点的浸入超曲面, 相应的 Möbius 位置向量是 $\tilde{Y}$.

设 $\left\{E_{i}, 1 \leqslant i \leqslant K\right\}$ 和 $\left.\left\{E_{i}, K+1 \leqslant i \leqslant m\right\}\right)$ 分别是 $\left(M_{1}, d \tilde{y}^{2}\right)$ 和 $S^{m-K}(r)$ 上的单位正交标 架场. 则 $\left\{E_{i}, 1 \leqslant i \leqslant m\right\}$ 是 $\left(\tilde{M}^{m}, g\right)$ 上的单位正交标架场, 其对偶标架场记为 $\left\{\omega^{i}, 1 \leqslant i \leqslant m\right\}$.

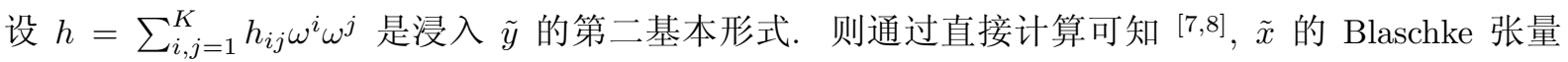
$A=\sum A_{i j} \omega^{i} \omega^{j}$ 和 Möbius 第二基本形式 $B=\sum B_{i j} \omega^{i} \omega^{j}$ 由下列式确定

$$
\begin{aligned}
& A_{i j}=-\left(\frac{1}{2 r^{2}}+\frac{1}{2} \lambda^{2}\right) \delta_{i j}-\lambda h_{i j}, \quad B_{i j}=h_{i j}+\lambda \delta_{i j}, \quad \text { 如果 } 1 \leqslant i, j \leqslant K ; \\
& A_{i j}=\left(\frac{1}{2 r^{2}}-\frac{1}{2} \lambda^{2}\right) \delta_{i j}, \quad B_{i j}=\lambda \delta_{i j}, \quad \text { 如果 } K+1 \leqslant i, j \leqslant m \text {; } \\
& A_{i j}=0, \quad B_{i j}=0, \quad \text { 其他. }
\end{aligned}
$$


因此, $\tilde{x}$ 具有常数 Blaschke 特征值当且仅当浸入 $\tilde{y}=\left(\tilde{y}_{0}, \tilde{y}_{1}\right): M_{1} \rightarrow H^{K+1}\left(-\frac{1}{r^{2}}\right)$ 是 (Euclid) 等参超 曲面. 此时, $\tilde{x}$ 的 Möbius 形式恒等于零, 因而是 Blaschke 等参超曲面.

注记 3.3 例 3.3 中的超曲面 $\tilde{x}$ 是 Blaschke 等参的. 它具有三个不同 Blaschke 特征值和四个不 同 Möbius 主曲率当且仅当相应的浸入 $\tilde{y}$ 是具有三个不同主曲率 $\mu_{1}, \mu_{2}, \mu_{3}$ 的非极小 (Euclid) 等参超 曲面, 且对某个 $i \in\{1,2,3\}$, 有 $\lambda \mu_{i}=-\frac{1}{r^{2}}$. 但是根据 Cartan 的结论 ${ }^{[23,24]}$, 这样的浸入 $\tilde{y}$ 是不存在的.

\section{$4 S^{6}$ 中具有四个不同 Blaschke 特征值的 Blaschke 等参超曲面}

要证明定理 1.4, 需要有关 $S^{6}$ 中 Möbius 等参超曲面的下述分类结果:

定理 4.1 ${ }^{[19]}$ 设 $x: M^{5} \rightarrow S^{6}$ 是单位球面 $S^{6}$ 中的 Möbius 等参超曲面. 则 $x$ 局部地 Möbius 等 价于下列超曲面之一:

(1) $S^{6}$ 中的标准环面 $S^{K}(r) \times S^{5-K}\left(\sqrt{1-r^{2}}\right)$, 其中 $r \in(0,1), K=1,2,3,4$;

(2) $\mathbb{R}^{6}$ 的标准柱面 $S^{K}(r) \times \mathbb{R}^{5-K}$ 在映射 $\sigma$ 下的像, 其中 $r>0, K=1,2,3,4$;

(3) $H^{6}$ 中的标准柱面 $S^{K}(r) \times H^{5-K}\left(-\frac{1}{1+r^{2}}\right)$ 在映射 $\tau$ 下的像, 其中 $r>0, K=1,2,3,4$;

(4) 例 3.1 中由某组参数 $p, q, r$ 确定的超曲面 $\operatorname{CSS}(p, q, r)$, 其中 $m=5$;

(5) 例 3.2 中由 $m=5, K=3$ 和某个 $r>0$ 确定的超曲面, 其中 $y_{1}$ 是 $S^{4}(r)$ 中具有三个不同主 曲率的 (Euclid) 等参超曲面;

(6) 例 3.2 中由 $m=5, K=4$ 和某个 $r>0$ 确定的超曲面, 其中 $y_{1}$ 是 $S^{5}(r)$ 中具有四个不同主 曲率的 (Euclid) 等参超曲面.

根据定理 1.2 和定理 1.3, $S^{6}$ 中具有不超过三个不同 Blaschke 特征值的 Blaschke 等参超曲面已 经有了完全的分类. 所以, 我们只需考虑 $S^{6}$ 中具有四个或五个不同 Blaschke 特征值的 Blaschke 等参 超曲面. 由于定理 4.1 和定理 1.1, 仅需证明如下的命题:

命题 $4.2 S^{6}$ 中具有四个不同 Blaschke 特征值的 Blaschke 等参超曲面一定是 Möbius 等参的.

为了证明命题 4.2 , 约定如下的指标取值范围:

$$
1 \leqslant a, b, c \leqslant 3, \quad 4 \leqslant \alpha, \beta, \gamma \leqslant 5 .
$$

设 $x: M^{5} \rightarrow S^{6}$ 是具有四个不同 Blaschke 特征值的 Blaschke 等参超曲面. 取关于 Möbius 度量 $g$ 的单位正交标架场 $\left\{E_{i}\right\}$, 使得 Blaschke 张量 $A$ 有下面的表达式:

$$
A=\sum A_{i j} \omega^{i} \omega^{j}, \quad A_{i j}=A_{i} \delta_{i j}
$$

其中的 $\left\{\omega^{i}\right\}$ 是 $\left\{E_{i}\right\}$ 的对偶标架场, $A_{i}, i=1, \ldots, 5$, 是 $A$ 的特征值. 不失一般性, 可以假设

$$
A_{1}<A_{2}<A_{3}, \quad A_{4}=A_{5}, \quad A_{1}, A_{2}, A_{3}, A_{4} \text { 互不相同. }
$$

不难看出, $E_{1}, E_{2}, E_{3}$ 可以取为在 $M$ 上 (至少在 $M$ 的一个单连通的开域内) 整体定义的向量场. 因此, 可以假设 $A_{123}$ 和 $B_{123}$ 也是整体定义的. 用 $V_{1} \rightarrow M$ 表示切丛 $T M$ 的由 $A$ 的对应于特征值 $A_{4}=A_{5}$ 的特征向量所构成的子丛. 则 $\left\{E_{4}, E_{5}\right\}$ 是 $V_{1}$ 的一个局部定义的单位正交标架场.

在上述的标架场 $\left\{E_{i}\right\}$ 下, $x$ 的 Möbius 第二基本形式可设为 $B=\sum B_{i j} \omega^{i} \omega^{j}$. 一般地, 不一定能 找到合适的单位正交标架场 $\left\{E_{i}\right\}$, 或等价地, 不一定能找到合适的 $\left\{E_{4}, E_{5}\right\}$, 使得 (4.2) 和

$$
B_{i j}=B_{i} \delta_{i j}, \quad \forall i, j,
$$


同时成立, 其中 $B_{1}, B_{2}, B_{3}, B_{4}, B_{5}$ 是 $x$ 的 Möbius 主曲率. 然而, 由于 Möbius 形式 $\Phi \equiv 0$, 从 (2.14) 易 知

$$
B_{a \alpha}=B_{\alpha a}=0, \quad B_{a b}=B_{a} \delta_{a b}, \quad 1 \leqslant a, b \leqslant 3, \quad 4 \leqslant \alpha \leqslant 5 .
$$

由此可见, $E_{1}, E_{2}$ 和 $E_{3}$ 也是 Möbius 第二基本形式 $B$ 的对应于 $B_{1}, B_{2}, B_{3}$ 的特征向量 (Möbius 主方 向). 而且, 对于任意的点 $p \in M$, 可以假定

$$
B_{45}(p)=0, \quad B_{4}(p)=B_{44}(p), \quad B_{5}(p)=B_{55}(p) .
$$

用 $M^{*}$ 表示满足下述条件的点 $p \in M$ 所构成的集合: 在 $p$ 点附近存在单位正交标架场 $\left\{E_{i}\right\}$ 使得 (4.2) 和 (4.4) 同时成立. 显然, $M^{*}$ 是 $M$ 的一个非空开子集. 后面的计算均假定在点 $p \in M^{*}$ 附近固 定了一个适当的局部标架场 $\left\{E_{i}\right\}$ 使得 (4.2) 和 (4.4) 恒成立.

设 $\omega_{i}^{j}$ 是度量 $g$ 的 Levi-Civita 联络关于 $\left\{E_{i}\right\}$ 的联络形式. 令 $\omega_{i}^{j}=\sum_{k} \Gamma_{i k}^{j} \omega^{k}$. 则 $\omega_{i}^{j}+\omega_{j}^{i}=0$, 或 等价地, $\Gamma_{i k}^{j}=-\Gamma_{j k}^{i}$.

利用 (2.15), (2.16) 和条件 $\Phi \equiv 0$ 可知, $A_{i j k}$ 和 $B_{i j k}$ 关于它们的下标是完全对称的. 因为 $A_{i}$ 都是 常数, 故对于任意的 $i, j$ 有 $A_{i j k} \omega^{k}=-A_{j} \omega_{i}^{j}-A_{i} \omega_{j}^{i}=\left(A_{i}-A_{j}\right) \Gamma_{i k}^{j} \omega^{k}$, 或等价地

$$
A_{i j k}=\left(A_{i}-A_{j}\right) \Gamma_{i k}^{j}=\left(A_{j}-A_{k}\right) \Gamma_{j i}^{k}=\left(A_{k}-A_{i}\right) \Gamma_{k j}^{i}, \quad \forall i, j, k .
$$

于是

$$
A_{i j i}=A_{j i i}=A_{i i j}=A_{a \alpha \beta}=0, \quad \forall i, j, a, \alpha, \beta .
$$

因此, 集合 $\left\{A_{i j k}, i \leqslant j \leqslant k\right\}$ 中有可能不恒为零的元素是 $A_{123}, A_{124}, A_{125}, A_{134}, A_{135}, A_{234}, A_{235}$. 除 此之外, (4.7) 式说明, 当 $A_{i} \neq A_{j}$ 时,

$$
\Gamma_{i k}^{j}=\frac{A_{i j k}}{A_{i}-A_{j}}, \text { 或等价地 } \omega_{i}^{j}=\sum_{k} \frac{A_{i j k}}{A_{i}-A_{j}} \omega^{k} .
$$

类似于 (4.7), 可由 (4.4) 得到

$$
B_{i j k}=\left(B_{i}-B_{j}\right) \Gamma_{i k}^{j}, \quad \forall i, j, k \text { 且 } i \neq j .
$$

根据 Gauss 方程 (2.13), 还可知

$$
R_{i j k l}=0, \text { 如果 }\{i, j, k, l\} \text { 中有三个互不相等. }
$$

引理 4.3 在定理的假设条件下, 有

$$
\begin{aligned}
& \frac{A_{124} A_{125}}{\left(A_{1}-A_{2}\right)\left(A_{2}-A_{4}\right)}=-\frac{A_{134} A_{135}}{\left(A_{1}-A_{3}\right)\left(A_{3}-A_{4}\right)}, \\
& \frac{A_{124} A_{125}}{\left(A_{1}-A_{2}\right)\left(A_{1}-A_{4}\right)}=\frac{A_{234} A_{235}}{\left(A_{2}-A_{3}\right)\left(A_{3}-A_{4}\right)} .
\end{aligned}
$$

证明 由 Ricci 恒等式和 (4.2) 式可知

$$
A_{i j k l}-A_{i j l k}=A_{p j} R_{i p k l}+A_{i p} R_{j p k l}=\left(A_{j}-A_{i}\right) R_{i j k l} .
$$

此式与 (4.11) 说明

$$
A_{i j k l}=A_{i j l k}, \quad i, j, k, l \text { 中有三个互不相等时. }
$$


利用 (4.8) 和 (4.9) 可得

$$
\begin{aligned}
\sum_{k} A_{451 k} \omega^{k}= & d A_{451}-\sum_{k} A_{k 51} \omega_{4}^{k}-\sum_{k} A_{4 k 1} \omega_{5}^{k}-\sum_{k} A_{45 k} \omega_{1}^{k} \\
= & -A_{251} \omega_{4}^{2}-A_{351} \omega_{4}^{3}-A_{421} \omega_{5}^{2}-A_{431} \omega_{5}^{3} \\
= & \left(-\frac{A_{124} A_{125}}{A_{4}-A_{2}}-\frac{A_{134} A_{135}}{A_{4}-A_{3}}-\frac{A_{124} A_{125}}{A_{5}-A_{2}}-\frac{A_{134} A_{135}}{A_{5}-A_{3}}\right) \omega^{1} \\
& +\left(-\frac{A_{234} A_{135}}{A_{4}-A_{3}}-\frac{A_{134} A_{235}}{A_{5}-A_{3}}\right) \omega^{2}+\left(-\frac{A_{234} A_{125}}{A_{4}-A_{2}}-\frac{A_{124} A_{235}}{A_{5}-A_{2}}\right) \omega^{3} \\
= & \left(\frac{2 A_{124} A_{125}}{A_{2}-A_{4}}+\frac{2 A_{134} A_{135}}{A_{3}-A_{4}}\right) \omega^{1} \\
& +\left(\frac{A_{234} A_{135}}{A_{3}-A_{4}}+\frac{A_{134} A_{235}}{A_{3}-A_{5}}\right) \omega^{2}+\left(\frac{A_{125} A_{234}}{A_{2}-A_{4}}+\frac{A_{124} A_{235}}{A_{2}-A_{4}}\right) \omega^{3} .
\end{aligned}
$$

因此

$$
A_{4511}=\frac{2 A_{124} A_{125}}{A_{2}-A_{4}}+\frac{2 A_{134} A_{135}}{A_{3}-A_{4}} .
$$

同样地, 有

$$
\begin{aligned}
\sum_{k} A_{114 k} \omega^{k}= & d A_{114}-\sum_{k} A_{k 14} \omega_{1}^{k}-\sum_{k} A_{1 k 4} \omega_{1}^{k}-\sum_{k} A_{11 k} \omega_{4}^{k} \\
= & -2 A_{124} \omega_{1}^{2}-2 A_{134} \omega_{1}^{3} \\
= & -\frac{2 A_{123} A_{134}}{A_{1}-A_{3}} \omega^{2}-\frac{2 A_{123} A_{124}}{A_{1}-A_{2}} \omega^{3}-\left(\frac{2 A_{124}^{2}}{A_{1}-A_{2}}+\frac{2 A_{134}^{2}}{A_{1}-A_{3}}\right) \omega^{4} \\
& -\left(\frac{2 A_{124} A_{125}}{A_{1}-A_{2}}+\frac{2 A_{134} A_{135}}{A_{1}-A_{3}}\right) \omega^{5} .
\end{aligned}
$$

这说明

$$
A_{1145}=-\left(\frac{2 A_{124} A_{125}}{A_{1}-A_{2}}+\frac{2 A_{134} A_{135}}{A_{1}-A_{3}}\right) .
$$

由 (4.15), $A_{1145}=A_{4511}$. 由此式结合 (4.16), (4.17) 即可得到 (4.12). 类似可得 (4.13).

引理 4.4 设 $(i j k)$ 是 $1,2,3$ 的一个排列. 则有

$$
\begin{aligned}
& R_{i j i j}=-\frac{2 A_{123}^{2}}{\left(A_{i}-A_{k}\right)\left(A_{j}-A_{k}\right)}-\frac{2\left(A_{i j 4}^{2}+A_{i j 5}^{2}\right)}{\left(A_{i}-A_{4}\right)\left(A_{j}-A_{4}\right)} \\
& R_{i 4 i 4}=-\frac{2 A_{i j 4}^{2}}{\left(A_{i}-A_{j}\right)\left(A_{4}-A_{j}\right)}-\frac{2 A_{i k 4}^{2}}{\left(A_{i}-A_{k}\right)\left(A_{4}-A_{k}\right)} \\
& R_{i 5 i 5}=-\frac{2 A_{i j 5}^{2}}{\left(A_{i}-A_{j}\right)\left(A_{5}-A_{j}\right)}-\frac{2 A_{i k 5}^{2}}{\left(A_{i}-A_{k}\right)\left(A_{5}-A_{k}\right)}
\end{aligned}
$$

证明 由 (4.14) 式,

$$
A_{i i j j}-A_{j j i i}=A_{i j i j}-A_{i j j i}=\left(A_{j}-A_{i}\right) R_{i j i j}
$$


利用 (4.8) 和 (4.9) 可知,

$$
\begin{aligned}
\sum_{l} A_{i i j l} \omega^{l}= & d A_{i i j}-2 \sum_{l} A_{i l j} \omega_{i}^{l}-\sum_{l} A_{i i l} \omega_{j}^{l} \\
= & -2 A_{i k j} \omega_{i}^{k}-2 A_{i 4 j} \omega_{i}^{4}-2 A_{i 5 j} \omega_{i}^{5} \\
= & -2 A_{i k j} \sum_{l} \frac{A_{i k l}}{A_{i}-A_{k}} \omega^{l}-2 A_{i 4 j} \sum_{l} \frac{A_{i 4 l}}{A_{i}-A_{4}} \omega^{l}-2 A_{i 5 j} \sum_{l} \frac{A_{i 5 l}}{A_{i}-A_{5}} \omega^{l} \\
= & -\frac{2 A_{i j k}^{2}}{A_{i}-A_{k}} \omega^{j}-\frac{2 A_{i j k} A_{i k 4}}{A_{i}-A_{k}} \omega^{4}-\frac{2 A_{i j k} A_{i k 5}}{A_{i}-A_{k}} \omega^{5} \\
& -\frac{2 A_{i 4 j}^{2}}{A_{i}-A_{4}} \omega^{j}-\frac{2 A_{i 4 j} A_{i k 4}}{A_{i}-A_{4}} \omega^{k}-\frac{2 A_{i 5 j}^{2}}{A_{i}-A_{5}} \omega^{j}-\frac{2 A_{i 5 j} A_{i k 5}}{A_{i}-A_{5}} \omega^{k} \\
= & -\left(\frac{2 A_{123}^{2}}{A_{i}-A_{k}}+\frac{2\left(A_{i j 4}^{2}+A_{i j 5}^{2}\right)}{A_{i}-A_{4}}\right) \omega^{j}+\cdots
\end{aligned}
$$

这蕴含着

$$
A_{i i j j}=-\frac{2 A_{123}^{2}}{A_{i}-A_{k}}-\frac{2\left(A_{i j 4}^{2}+A_{i j 5}^{2}\right)}{A_{i}-A_{4}} .
$$

类似地,

$$
A_{j j i i}=-\frac{2 A_{123}^{2}}{A_{j}-A_{k}}-\frac{2\left(A_{i j 4}^{2}+A_{i j 5}^{2}\right)}{A_{j}-A_{4}} .
$$

于是, 从 (4.21)-(4.23) 可得到 (4.18) 式.

另一方面, 容易看出

$$
A_{i i 44}=-\frac{2 A_{i j 4}^{2}}{A_{i}-A_{j}}-\frac{2 A_{i k 4}^{2}}{A_{i}-A_{k}}, \quad A_{44 i i}=-\frac{2 A_{i j 4}^{2}}{A_{4}-A_{j}}-\frac{2 A_{i k 4}^{2}}{A_{4}-A_{k}} .
$$

由 (4.21) 和 (4.24) 容易得到 (4.19). (4.20) 可类似得到.

引理 4.5 对于任意不同的 $i, j \in\{1,2,3\}$, 如果在一点 $p \in M^{*}$ 处 $A_{123} A_{i j \alpha} \neq 0$, 则在 $p$ 点附近, 对于任意的 $\beta \neq \alpha$ 成立 $A_{12 \beta}=A_{13 \beta}=A_{23 \beta}=0$.

证明 不妨设 $(i, j, \alpha)=(1,2,4)$. 只需证明, $A_{123} A_{124}(p) \neq 0$ 蕴含着在 $p$ 点 $A_{125}=A_{135}=A_{235}=$ 0.

如若不然, 则在 $p$ 点的一个开邻域 $U$ 内可设 $A_{125} \neq 0$. 根据 (4.7), (4.10) 和 $A_{i j k}$ 和 $B_{i j k}$ 的对称 性,

$$
\begin{aligned}
& A_{123}=\left(A_{1}-A_{2}\right) \Gamma_{13}^{2}=\left(A_{2}-A_{3}\right) \Gamma_{21}^{3}=\left(A_{3}-A_{1}\right) \Gamma_{32}^{1}, \\
& B_{123}=\left(B_{1}-B_{2}\right) \Gamma_{13}^{2}=\left(B_{2}-B_{3}\right) \Gamma_{21}^{3}=\left(B_{3}-B_{1}\right) \Gamma_{32}^{1}, \\
& A_{124}=\left(A_{1}-A_{2}\right) \Gamma_{14}^{2}=\left(A_{2}-A_{4}\right) \Gamma_{21}^{4}=\left(A_{4}-A_{1}\right) \Gamma_{42}^{1}, \\
& B_{124}=\left(B_{1}-B_{2}\right) \Gamma_{14}^{2}=\left(B_{2}-B_{4}\right) \Gamma_{21}^{4}=\left(B_{4}-B_{1}\right) \Gamma_{42}^{1}, \\
& A_{125}=\left(A_{1}-A_{2}\right) \Gamma_{15}^{2}=\left(A_{2}-A_{5}\right) \Gamma_{21}^{5}=\left(A_{5}-A_{1}\right) \Gamma_{52}^{1}, \\
& B_{125}=\left(B_{1}-B_{2}\right) \Gamma_{15}^{2}=\left(B_{2}-B_{5}\right) \Gamma_{21}^{5}=\left(B_{5}-B_{1}\right) \Gamma_{52}^{1} .
\end{aligned}
$$


再从 (4.25)-(4.30), 容易看出

$$
\begin{aligned}
& \frac{B_{123}}{A_{123}}=\frac{B_{1}-B_{2}}{A_{1}-A_{2}}=\frac{B_{2}-B_{3}}{A_{2}-A_{3}}=\frac{B_{3}-B_{1}}{A_{3}-A_{1}}, \\
& \frac{B_{124}}{A_{124}}=\frac{B_{1}-B_{2}}{A_{1}-A_{2}}=\frac{B_{2}-B_{4}}{A_{2}-A_{4}}=\frac{B_{4}-B_{1}}{A_{4}-A_{1}}, \\
& \frac{B_{125}}{A_{125}}=\frac{B_{1}-B_{2}}{A_{1}-A_{2}}=\frac{B_{2}-B_{5}}{A_{2}-A_{5}}=\frac{B_{5}-B_{1}}{A_{5}-A_{1}} .
\end{aligned}
$$

由于 $A_{123} A_{124} A_{125} \neq 0$, 所以, 如果 $B_{123}, B_{124}, B_{125}$ 中有一个在某一点等于零, 则其余两个在同 一点也必为零. 于是在该点有 $B_{1}=\cdots=B_{5}$, 与 $(2.12)$ 式矛盾. 可见在 $U$ 上 $B_{123} B_{124} B_{125} \neq 0$. 因此 存在函数 $\lambda \neq 0$, 使得

$$
\frac{B_{1}-B_{2}}{A_{1}-A_{2}}=\frac{B_{2}-B_{3}}{A_{2}-A_{3}}=\frac{B_{1}-B_{3}}{A_{1}-A_{3}}=\frac{B_{2}-B_{4}}{A_{2}-A_{4}}=\frac{B_{2}-B_{5}}{A_{2}-A_{5}}=\frac{B_{1}-B_{4}}{A_{1}-A_{4}}=\frac{B_{1}-B_{5}}{A_{1}-A_{5}}=-\frac{1}{\lambda} .
$$

因而, 存在另一个函数 $\mu$ 使得

$$
A_{1}+\lambda B_{1}=A_{2}+\lambda B_{2}=A_{3}+\lambda B_{3}=A_{4}+\lambda B_{4}=A_{5}+\lambda B_{5}=\mu .
$$

这说明 $B_{4}=B_{5}$, 且

$$
A+\lambda B-\mu g=0 .
$$

根据文献 [3] 中的结论知, $\lambda$ 和 $\mu$ 是常数, 并且 $x: U \rightarrow S^{6}$ 局部地 Möbius 等价于下列超曲面中的一 个:

(1) 具有常平均曲率和常数量曲率的浸入超曲面 $x: U \rightarrow S^{6}$;

(2) 具有常平均曲率和常数量曲率的浸入超曲面 $x: U \rightarrow \mathbb{R}^{6}$ 在映射 $\sigma$ 下的像;

(3) 具有常平均曲率和常数量曲率的浸入超曲面 $x: U \rightarrow H^{6}$ 在映射 $\tau$ 下的像.

注意到上述超曲面 $x$ 的 Möbius 因子均为常数, 并且由 (4.32), $B_{1}, B_{2}, B_{3}, B_{4}=B_{5}$ 均为常数. 所 以, $x$ 是具有四个不同主曲率的 (Euclid) 等参超曲面. 但是根据有关等参超曲面的经典结果 (比如: 对 于单位球面中具有四个不同主曲率的等参超曲面可以参见文献 [23]; 对于 Euclid 空间和双曲空间中的 等参超曲面则可以分别参见文献 [24] 和 [22]), 6 维单位球面 $S^{6}$ 或 Euclid 空间 $\mathbb{R}^{n}$ 或双曲空间 $H^{n}$ 中 均没有具有四个不同主曲率的等参超曲面. 这个矛盾说明在 $p$ 附近有 $A_{125} \equiv 0$.

类似可证, 在 $p$ 附近 $A_{135}=A_{235} \equiv 0$ 成立.

引理 4.6 在 $M^{*}$ 上,

$$
A_{124} A_{125}=A_{134} A_{135}=A_{234} A_{235}=0 .
$$

特别地, 集合

$$
\left\{A_{124}, A_{125}, A_{134}, A_{135}, A_{234}, A_{235}\right\}
$$

中至多有三个元素不为零.

证明 如果 $A_{124} A_{125}, A_{134} A_{135}, A_{234} A_{235}$ 中有一个在某点 $p \in M^{*}$ 处不为零, 比如 $A_{124} A_{125}(p) \neq$ 0 , 则 $A_{124} A_{125}$ 在 $p$ 的某一开邻域 $U$ 内恒不为零. 由引理 4.3, 在 $U$ 上 $A_{134} A_{135} A_{234} A_{235} \neq 0$. 由此结 合引理 4.5 的证明可知, 有 $U$ 上的函数 $\lambda \neq 0$ 和 $\mu$, 使得 (4.32) 成立. 再用引理 4.5 后边的推理又可 导出矛盾.

考虑如下的两种情形: 情形 I. 在 $M^{*}$ 上 $A_{123} \equiv 0$; 情形 II. 在某一开集 $U \subset M^{*}$ 上, $A_{123} \neq 0$. 
对于情形 I, 有下面的两个引理:

引理 4.7 如果在 $M^{*}$ 上, $A_{123} \equiv 0$, 则 $\left\{A_{124}, A_{125}, A_{134}, A_{135}, A_{234}, A_{235}\right\}$ 恰有两个非零元素.

证明 根据具有平行 Blaschke 张量的超曲面的分类定理 ([6]), $x: M^{5} \rightarrow S^{6}$ 有四个不同 Blaschke 特征值的假设说明 $x$ 的 Blaschke 张量一定不是平行的. 于是在 $A_{124}, A_{125}, A_{134}, A_{135}, A_{234}, A_{235}$ 中 至少有一不为零. 不失一般性, 设 $A_{124} \neq 0$. 则由引理 4.6 知, $A_{125}=0$.

如果引理的结论不对, 则有下列两种情形:

(1) $A_{134}, A_{135}, A_{234}, A_{235}$ 恒为零. 根据 (2.13), (4.2), (4.4) 和引理 4.4, 在此情形下, 如下等式成立:

$$
\begin{aligned}
& R_{1331}=B_{1} B_{3}+A_{1}+A_{3}=0, \\
& R_{2332}=B_{2} B_{3}+A_{2}+A_{3}=0, \\
& R_{2442}=B_{2} B_{4}+A_{2}+A_{4}=\frac{2 A_{124}^{2}}{\left(A_{2}-A_{1}\right)\left(A_{4}-A_{1}\right)}, \\
& R_{2552}=B_{2} B_{5}+A_{2}+A_{5}=0, \\
& R_{3443}=B_{3} B_{4}+A_{3}+A_{4}=0, \\
& R_{3553}=B_{3} B_{5}+A_{3}+A_{5}=0 .
\end{aligned}
$$

由 (4.33) 和 (4.34), $B_{3} \neq 0$. 结合 (4.37) 和 (4.38) 可知 $B_{4}=B_{5}$. 再由 (4.35) 和 (4.36) 可得 $A_{124}=0$, 与假设矛盾.

(2) $\left\{A_{134}, A_{135}, A_{234}, A_{235}\right\}$ 中恰好有两个元素不为零. 不失一般性可设 $A_{134} \neq 0$. 那么, 由引理 4.6 得, $A_{135}=0$. 因而有 $A_{234} \neq 0$ 或 $A_{235} \neq 0$.

如果 $A_{234} \neq 0$, 则有 $A_{235}=0$. 由 (4.8), (4.9), 以及 $A_{123}=A_{125}=A_{135}=A_{235}=0$, 可以看出

$$
\begin{aligned}
& \omega_{1}^{2}= \sum_{k} \frac{A_{12 k}}{A_{1}-A_{2}} \omega^{k}=\frac{A_{124}}{A_{1}-A_{2}} \omega^{4}, \quad \omega_{1}^{3}=\sum_{k} \frac{A_{13 k}}{A_{1}-A_{3}} \omega^{k}=\frac{A_{134}}{A_{1}-A_{2}} \omega^{4}, \\
& \omega_{1}^{4}=\sum_{k} \frac{A_{14 k}}{A_{1}-A_{4}} \omega^{k}=\frac{A_{142}}{A_{1}-A_{4}} \omega^{2}+\frac{A_{143}}{A_{1}-A_{4}} \omega^{3}, \\
& \omega_{1}^{5}=\sum_{k} \frac{A_{15 k}}{A_{1}-A_{5}} \omega^{k}=0, \quad \omega_{2}^{3}=\sum_{k} \frac{A_{23 k}}{A_{2}-A_{3}} \omega^{k}=\frac{A_{234}}{A_{2}-A_{3}} \omega^{4}, \\
& \omega_{2}^{4}=\sum_{k} \frac{A_{24 k}}{A_{2}-A_{4}} \omega^{k}=\frac{A_{241}}{A_{2}-A_{4}} \omega^{1}+\frac{A_{243}}{A_{2}-A_{4}} \omega^{3}, \\
& \omega_{3}^{4}=\sum_{k} \frac{A_{34 k}}{A_{3}-A_{4}} \omega^{k}=\frac{A_{341}}{A_{3}-A_{4}} \omega^{1}+\frac{A_{342}}{A_{3}-A_{4}} \omega^{2}, \\
& \omega_{2}^{5}=\sum_{k} \frac{A_{25 k}}{A_{2}-A_{5}} \omega^{k}=0, \\
& d \omega^{4}=\omega^{1} \wedge \omega_{1}^{4}+\omega^{2} \wedge \omega_{2}^{4}+\omega^{3} \wedge \omega_{3}^{4}+\omega^{5} \wedge \omega_{5}^{4} \\
&=\omega^{1} \wedge\left(\frac{A_{142}}{A_{1}-A_{4}} \omega^{2}+\frac{A_{143}}{A_{1}-A_{4}} \omega^{3}\right)+\omega^{2} \wedge\left(\frac{A_{241}}{A_{2}-A_{4}} \omega^{1}+\frac{A_{243}}{A_{2}-A_{4}} \omega^{3}\right) \\
&+\omega^{3} \wedge\left(\frac{A_{341}}{A_{3}-A_{4}} \omega^{1}+\frac{A_{342}}{A_{3}-A_{4}} \omega^{2}\right)+\omega^{5} \wedge \omega_{5}^{4} \\
&= \\
& \quad\left(\frac{A_{134}}{A_{1}-A_{4}} \frac{A_{134}}{A_{3}-A_{4}}\right) \omega^{1} \wedge \omega^{3}+\left(\frac{A_{234}}{A_{2}-A_{4}}-\frac{A_{234}}{A_{3}-A_{4}}\right) \omega^{2} \wedge \omega^{3}+\cdots
\end{aligned}
$$


再由 (4.11) 式,

$$
\begin{aligned}
R_{1212} \omega^{1} \wedge \omega^{2}= & \frac{1}{2} \sum_{i, j} R_{12 i j} \omega^{i} \wedge \omega^{j}=d \omega_{1}^{2}-\sum_{k} \omega_{1}^{k} \wedge \omega_{k}^{2} \\
= & \frac{d A_{124}}{A_{1}-A_{2}} \wedge \omega^{4}+\frac{A_{124}}{A_{1}-A_{2}} d \omega^{4} \\
& +\left(\frac{A_{142}}{A_{1}-A_{4}} \omega^{2}+\frac{A_{143}}{A_{1}-A_{4}} \omega^{3}\right) \wedge\left(\frac{A_{124}}{A_{2}-A_{4}} \omega^{1}+\frac{A_{234}}{A_{2}-A_{4}} \omega^{3}\right) \\
= & \frac{A_{124}}{A_{1}-A_{2}} \cdot\left(\frac{A_{134}}{A_{1}-A_{4}}-\frac{A_{134}}{A_{3}-A_{4}}\right) \omega^{1} \wedge \omega^{3} \\
& +\frac{A_{124}}{A_{1}-A_{2}} \cdot\left(\frac{A_{234}}{A_{2}-A_{4}}-\frac{A_{234}}{A_{3}-A_{4}}\right) \omega^{2} \wedge \omega^{3} \\
& -\frac{A_{124}}{A_{2}-A_{4}} \cdot \frac{A_{134}}{A_{1}-A_{4}} \omega^{1} \wedge \omega^{3}+\frac{A_{124}}{A_{1}-A_{4}} \cdot \frac{A_{234}}{A_{2}-A_{4}} \omega^{2} \wedge \omega^{3}+\cdots \\
= & \left(\frac{A_{124} A_{134}}{\left(A_{1}-A_{2}\right)\left(A_{1}-A_{4}\right)}-\frac{A_{124} A_{134}}{\left(A_{1}-A_{2}\right)\left(A_{3}-A_{4}\right)}-\frac{A_{124} A_{134}}{\left(A_{1}-A_{4}\right)\left(A_{2}-A_{4}\right)}\right) \omega^{1} \wedge \omega^{3} \\
& +\left(\frac{A_{124} A_{234}}{\left(A_{1}-A_{2}\right)\left(A_{2}-A_{4}\right)}-\frac{A_{124} A_{234}}{\left(A_{1}-A_{2}\right)\left(A_{3}-A_{4}\right)}+\frac{A_{124} A_{234}}{\left(A_{1}-A_{4}\right)\left(A_{2}-A_{4}\right)}\right) \omega^{2} \wedge \omega^{3} \\
& +\cdots,
\end{aligned}
$$

其中 “...” 代表若干不含 $\omega^{1} \wedge \omega^{3}$ 和 $\omega^{2} \wedge \omega^{3}$ 的项. 分别比较上式两边 $\omega^{1} \wedge \omega^{3}$ 和 $\omega^{2} \wedge \omega^{3}$ 的系数可以 得到

$$
\begin{aligned}
& \frac{1}{\left(A_{1}-A_{2}\right)\left(A_{1}-A_{4}\right)}-\frac{1}{\left(A_{1}-A_{2}\right)\left(A_{3}-A_{4}\right)}-\frac{1}{\left(A_{1}-A_{4}\right)\left(A_{2}-A_{4}\right)}=0, \\
& \frac{1}{\left(A_{1}-A_{2}\right)\left(A_{2}-A_{4}\right)}-\frac{1}{\left(A_{1}-A_{2}\right)\left(A_{3}-A_{4}\right)}+\frac{1}{\left(A_{1}-A_{4}\right)\left(A_{2}-A_{4}\right)}=0 .
\end{aligned}
$$

因而有

$$
\frac{1}{\left(A_{1}-A_{4}\right)\left(A_{2}-A_{4}\right)}=0
$$

这是不可能的!

如果 $A_{235} \neq 0$, 则 $A_{234}=0$. 由 (4.8), (4.9), 以及 $A_{123}=A_{125}=A_{135}=A_{234}=0$, 可以看出

$$
\begin{aligned}
& \omega_{1}^{2}=\frac{A_{124}}{A_{1}-A_{2}} \omega^{4}, \quad \omega_{1}^{3}=\frac{A_{134}}{A_{1}-A_{2}} \omega^{4}, \quad \omega_{1}^{4}=\frac{A_{124}}{A_{1}-A_{4}} \omega^{2}+\frac{A_{134}}{A_{1}-A_{4}} \omega^{3}, \\
& \omega_{1}^{5}=0, \quad \omega_{2}^{3}=\frac{A_{235}}{A_{2}-A_{3}} \omega^{5}, \quad \omega_{2}^{4}=\frac{A_{124}}{A_{2}-A_{4}} \omega^{1}, \\
& \omega_{3}^{4}=\frac{A_{134}}{A_{3}-A_{4}} \omega^{1}, \quad \omega_{2}^{5}=\frac{A_{235}}{A_{2}-A_{5}} \omega^{3}, \quad \omega_{3}^{5}=\frac{A_{235}}{A_{3}-A_{5}} \omega^{2}, \\
& d \omega^{4}=\left(\frac{A_{134}}{A_{1}-A_{4}}-\frac{A_{134}}{A_{3}-A_{4}}\right) \omega^{1} \wedge \omega^{3}+\left(\frac{A_{124}}{A_{1}-A_{4}}-\frac{A_{124}}{A_{2}-A_{4}}\right) \omega^{1} \wedge \omega^{2}+\cdots,
\end{aligned}
$$

其中 “...”代表若干不含 $\omega^{1} \wedge \omega^{3}$ 和 $\omega^{1} \wedge \omega^{2}$ 的项. 由 (4.11) 进行直接计算得

$$
\begin{aligned}
R_{1212} \omega^{1} \wedge \omega^{2}= & \left(\frac{A_{124} A_{134}}{\left(A_{1}-A_{2}\right)\left(A_{1}-A_{4}\right)}-\frac{A_{124} A_{134}}{\left(A_{1}-A_{2}\right)\left(A_{3}-A_{4}\right)}\right. \\
& \left.-\frac{A_{124} A_{134}}{\left(A_{1}-A_{4}\right)\left(A_{2}-A_{4}\right)}\right) \omega^{1} \wedge \omega^{3}+\cdots,
\end{aligned}
$$




$$
\begin{aligned}
R_{1313} \omega^{1} \wedge \omega^{3}= & \left(\frac{A_{124} A_{134}}{\left(A_{1}-A_{2}\right)\left(A_{1}-A_{4}\right)}-\frac{A_{124} A_{134}}{\left(A_{1}-A_{2}\right)\left(A_{2}-A_{4}\right)}\right. \\
& \left.-\frac{A_{124} A_{134}}{\left(A_{1}-A_{4}\right)\left(A_{3}-A_{4}\right)}\right) \omega^{1} \wedge \omega^{2}+\cdots
\end{aligned}
$$

(4.39) 和 (4.40) 中的两处 “...” 分别表示一些不含 $\omega^{1} \wedge \omega^{3}$ 和 $\omega^{1} \wedge \omega^{2}$ 的项. 在 (4.39) 和 (4.40) 的两 边分别比较 $\omega^{1} \wedge \omega^{3}$ 和 $\omega^{1} \wedge \omega^{2}$ 的系数, 可得

$$
\begin{aligned}
& \frac{1}{\left(A_{1}-A_{2}\right)\left(A_{1}-A_{4}\right)}-\frac{1}{\left(A_{1}-A_{2}\right)\left(A_{3}-A_{4}\right)}-\frac{1}{\left(A_{1}-A_{4}\right)\left(A_{2}-A_{4}\right)}=0, \\
& \frac{1}{\left(A_{1}-A_{2}\right)\left(A_{1}-A_{4}\right)}-\frac{1}{\left(A_{1}-A_{2}\right)\left(A_{2}-A_{4}\right)}-\frac{1}{\left(A_{1}-A_{4}\right)\left(A_{3}-A_{4}\right)}=0 .
\end{aligned}
$$

这说明

$$
\frac{\left(A_{2}-A_{3}\right)\left(A_{2}-A_{4}\right)}{\left(A_{1}-A_{2}\right)\left(A_{1}-A_{4}\right)\left(A_{2}-A_{4}\right)\left(A_{3}-A_{4}\right)}=0
$$

与假设矛盾.

引理 4.8 如果在 $M^{*}$ 上 $A_{123} \equiv 0$, 则 $x: M^{*} \rightarrow S^{6}$ 是 Möbius 等参超曲面.

证明 由引理 4.7, $A_{124}, A_{125}, A_{134}, A_{135}, A_{234}, A_{235}$ 中恰好有两个不为零. 根据引理 4.6, 只需考 虑以下两种情形: (1) $A_{124} A_{134} \neq 0$, (2) $A_{124} A_{235} \neq 0$. 下面将说明: 情形 (1) 蕴含着 $x$ 是 Möbius 等参 的; 而情形 (2) 则不会出现.

(1) 如果 $A_{124} A_{134} \neq 0$, 则在某一开集 $U \subset M^{*}$ 内 $A_{125}=A_{135}=A_{234}=A_{235}=0$ 恒成立. 于是 由 (2.13), (4.2), (4.4) 和引理 4.4,

$$
\begin{array}{ll}
R_{2332}=B_{2} B_{3}+A_{2}+A_{3}=0, & R_{1551}=B_{1} B_{5}+A_{1}+A_{5}=0, \\
R_{2552}=B_{2} B_{5}+A_{2}+A_{5}=0, & R_{3553}=B_{3} B_{5}+A_{3}+A_{5}=0,
\end{array}
$$

由于 $A_{1}, A_{2}, A_{3}$ 和 $A_{5}$ 彼此不同, $B_{2} B_{3} B_{5} \neq 0$. 特别地, $A_{2}+A_{5} \neq 0$.

此外, 由 (4.41) 和 (4.42) 的第一式可得

$$
B_{3}=\frac{A_{2}+A_{3}}{A_{2}+A_{5}} B_{5}
$$

将 (4.43) 代入 (4.42) 的第二式得

$$
B_{5}^{2}=-\frac{\left(A_{2}+A_{5}\right)\left(A_{3}+A_{5}\right)}{A_{2}+A_{3}}=\text { const. }
$$

结合 (4.41) 的第二式, (4.42), 以及 $B_{5} \neq 0$, 可以推知 $B_{1}, B_{2}$ 和 $B_{3}$ 也是常数. 因而,

$$
B_{4}=-\left(B_{1}+B_{2}+B_{3}+B_{5}\right)
$$

是常数. 所以, Möbius 主曲率 $B_{1}, \ldots, B_{5}$ 均为常数, 因而 $x$ 是 Möbius 等参超曲面.

(2) 如果 $A_{124} A_{235} \neq 0$, 则 $A_{123}=A_{125}=A_{134}=A_{135}=A_{234}=0$ 在某个开集 $U \subset M^{*}$ 上恒成立. 由 (4.9) 式,

$$
\omega_{1}^{3}=\omega_{1}^{5}=\omega_{3}^{4}=0, \quad \omega_{2}^{3}=\frac{A_{235}}{A_{2}-A_{3}} \omega^{5}, \quad \omega_{2}^{4}=\frac{A_{124}}{A_{2}-A_{4}} \omega^{1}, \quad \omega_{3}^{5}=\frac{A_{235}}{A_{3}-A_{5}} \omega^{2} .
$$


因此

$$
\begin{aligned}
R_{3434} \omega^{3} \wedge \omega^{4} & =d \omega_{3}^{4}+\omega_{2}^{3} \wedge \omega_{2}^{4}+\omega_{3}^{5} \wedge \omega_{4}^{5} \\
& =-\frac{A_{124} A_{235}}{\left(A_{2}-A_{3}\right)\left(A_{2}-A_{4}\right)} \omega^{1} \wedge \omega^{5}+\frac{A_{235}}{A_{3}-A_{5}} \omega^{2} \wedge \omega_{4}^{5}
\end{aligned}
$$

比较上式两边 $\omega^{1} \wedge \omega^{5}$ 的系数可得

$$
\frac{A_{124} A_{235}}{\left(A_{2}-A_{3}\right)\left(A_{2}-A_{4}\right)}=0
$$

与假设矛盾. 可见, 情形 (2) 是不可能出现的.

对于情形 II, 有如下的四个引理:

引理 4.9 如果在某个开集 $U \subset M^{*}$ 上有 $A_{123} \neq 0$, 则在集合 $\left\{A_{124}, A_{125}, A_{134}, A_{135}, A_{234}, A_{235}\right\}$ 中不会恰好有两个元素在 $U$ 上不为零.

证明 根据引理 4.5, 只需考虑情形: $A_{123} A_{124} A_{134} \neq 0$, 并且 $A_{125}=A_{135}=A_{234}=A_{235}=0$. 此 时有

$$
\begin{aligned}
\omega_{i}^{5}= & 0, \quad i=1,2,3,4 \\
\omega_{1}^{2}= & \frac{A_{123}}{A_{1}-A_{2}} \omega^{3}+\frac{A_{124}}{A_{1}-A_{2}} \omega^{4}, \quad \omega_{2}^{3}=\frac{A_{123}}{A_{2}-A_{3}} \omega^{1}, \\
\omega_{1}^{3}= & \frac{A_{123}}{A_{1}-A_{3}} \omega^{2}+\frac{A_{134}}{A_{1}-A_{3}} \omega^{4}, \quad \omega_{2}^{4}=\frac{A_{124}}{A_{2}-A_{4}} \omega^{1}, \\
\omega_{1}^{4}= & \frac{A_{124}}{A_{1}-A_{4}} \omega^{2}+\frac{A_{134}}{A_{1}-A_{4}} \omega^{3}, \quad \omega_{3}^{4}=\frac{A_{134}}{A_{3}-A_{4}} \omega^{1}, \\
d \omega^{1}= & -\omega^{2} \wedge\left(\frac{A_{123}}{A_{1}-A_{2}} \omega^{3}+\frac{A_{124}}{A_{1}-A_{2}} \omega^{4}\right)-\omega^{3} \wedge\left(\frac{A_{123}}{A_{1}-A_{3}} \omega^{2}+\frac{A_{134}}{A_{1}-A_{3}} \omega^{4}\right) \\
& -\omega^{4} \wedge\left(\frac{A_{124}}{A_{1}-A_{4}} \omega^{2}+\frac{A_{134}}{A_{1}-A_{4}} \omega^{3}\right) \\
= & \left(\frac{A_{124}}{A_{1}-A_{4}}-\frac{A_{124}}{A_{1}-A_{2}}\right) \omega^{2} \wedge \omega^{4}+\left(\frac{A_{134}}{A_{1}-A_{4}}-\frac{A_{134}}{A_{1}-A_{3}}\right) \omega^{3} \wedge \omega^{4}+\cdots,
\end{aligned}
$$

其中 “...” 表示若干不含 $\omega^{2} \wedge \omega^{4}$ 和 $\omega^{3} \wedge \omega^{4}$ 的项. 因此

$$
\begin{aligned}
R_{2323} \omega^{2} \wedge \omega^{3}= & d \omega_{2}^{3}-\omega_{2}^{1} \wedge \omega_{1}^{3}-\omega_{2}^{4} \wedge \omega_{4}^{3}-\omega_{2}^{5} \wedge \omega_{5}^{3} \\
= & \frac{d A_{123}}{A_{2}-A_{3}} \wedge \omega^{1}+\frac{A_{123}}{A_{2}-A_{3}}\left(\left(\frac{A_{124}}{A_{1}-A_{4}}-\frac{A_{124}}{A_{1}-A_{2}}\right) \omega^{2} \wedge \omega^{4}\right. \\
& \left.+\left(\frac{A_{134}}{A_{1}-A_{4}}-\frac{A_{134}}{A_{1}-A_{3}}\right) \omega^{3} \wedge \omega^{4}+\cdots\right) \\
& +\left(\frac{A_{123}}{A_{1}-A_{2}} \omega^{3}+\frac{A_{124}}{A_{1}-A_{2}} \omega^{4}\right) \wedge\left(\frac{A_{123}}{A_{1}-A_{3}} \omega^{2}+\frac{A_{134}}{A_{1}-A_{3}} \omega^{4}\right) \\
= & \left(\frac{A_{123} A_{124}}{\left(A_{1}-A_{4}\right)\left(A_{2}-A_{3}\right)}-\frac{A_{123} A_{124}}{\left(A_{1}-A_{2}\right)\left(A_{2}-A_{3}\right)}-\frac{A_{123} A_{124}}{\left(A_{1}-A_{2}\right)\left(A_{1}-A_{3}\right)}\right) \omega^{2} \wedge \omega^{4} \\
& +\left(\frac{A_{123} A_{134}}{\left(A_{1}-A_{4}\right)\left(A_{2}-A_{3}\right)}-\frac{A_{123} A_{134}}{\left(A_{1}-A_{3}\right)\left(A_{2}-A_{3}\right)}+\frac{A_{123} A_{134}}{\left(A_{1}-A_{2}\right)\left(A_{1}-A_{3}\right)}\right) \omega^{3} \wedge \omega^{4} \\
& +\cdots .
\end{aligned}
$$


分别比较上式两边 $\omega^{2} \wedge \omega^{4}$ 和 $\omega^{3} \wedge \omega^{4}$ 的系数, 并注意到 $A_{123} A_{124} A_{134} \neq 0$, 即得

$$
\begin{aligned}
& \frac{1}{\left(A_{1}-A_{4}\right)\left(A_{2}-A_{3}\right)}-\frac{1}{\left(A_{1}-A_{2}\right)\left(A_{2}-A_{3}\right)}-\frac{1}{\left(A_{1}-A_{2}\right)\left(A_{1}-A_{3}\right)}=0, \\
& \frac{1}{\left(A_{1}-A_{4}\right)\left(A_{2}-A_{3}\right)}-\frac{1}{\left(A_{1}-A_{3}\right)\left(A_{2}-A_{3}\right)}+\frac{1}{\left(A_{1}-A_{2}\right)\left(A_{1}-A_{3}\right)}=0 .
\end{aligned}
$$

由此可得

$$
\frac{1}{\left(A_{1}-A_{2}\right)\left(A_{1}-A_{3}\right)}=0
$$

矛盾.

引理 4.10 如果在某个开集 $U \subset M^{*}$ 上, $A_{123} \neq 0$, 并且 $A_{124}, A_{125}, A_{134}, A_{135}, A_{234}, A_{235}$ 全部 为零, 则 $x: U \rightarrow S^{6}$ 是 Möbius 等参超曲面.

证明 从 (2.13), (4.2), (4.4), 引理 4.4, 以及 $A_{124}=A_{125}=A_{134}=A_{135}=A_{234}=A_{235}=0$, 可知

$$
\begin{aligned}
& R_{1441}=B_{1} B_{4}+A_{1}+A_{4}=0, \quad R_{1551}=B_{1} B_{5}+A_{1}+A_{5}=0, \\
& R_{2442}=B_{2} B_{4}+A_{2}+A_{4}=0, \quad R_{2552}=B_{2} B_{5}+A_{2}+A_{5}=0, \\
& R_{3443}=B_{3} B_{4}+A_{3}+A_{4}=0, \quad R_{3553}=B_{3} B_{5}+A_{3}+A_{5}=0,
\end{aligned}
$$

易见, $B_{4} B_{5} \neq 0$.

我们断言: 在 $U$ 上 $B_{4} \equiv B_{5}$. 事实上, 如果在某一点 $p \in U, B_{4} \neq B_{5}$, 则由 (4.44)-(4.46) 可知, 在 $p$ 点成立 $B_{1}=B_{2}=B_{3}=0$. 结合 (4.44) 和 (4.45) 即得 $A_{1}=A_{2}$. 这是不可能的!

另一方面, 从 (4.44)-(4.46), 以及 $B_{4} \equiv B_{5}$ 可以看出

$$
2 B_{4}^{2}=-\left(B_{1}+B_{2}+B_{3}\right) B_{4}=A_{1}+A_{2}+A_{3}+3 A_{4}=\text { const. }
$$

由于 $B_{4} \neq 0$, 上式说明 $B_{1}, B_{2}, B_{3}$ 也是常数. 故所有的 Möbius 主曲率都是常数.

引理 4.11 如果在某个开集 $U \subset M^{*}$ 上, $A_{123} \neq 0$, 并且 $\left\{A_{124}, A_{125}, A_{134}, A_{135}, A_{234}, A_{235}\right\}$ 中 恰好有一个元素不为零, 则 $x: U \rightarrow S^{6}$ 是 Möbius 等参超曲面.

证明 不失一般性, 可设 $A_{124} \neq 0$. 则 $A_{125}=A_{134}=A_{135}=A_{234}=A_{235}=0$. 这说明

$$
\begin{aligned}
& R_{1441}=B_{1} B_{4}+A_{1}+A_{4}=\frac{2 A_{124}^{2}}{\left(A_{1}-A_{2}\right)\left(A_{4}-A_{2}\right)}, \\
& R_{1551}=B_{1} B_{5}+A_{1}+A_{5}=0, \quad R_{2552}=B_{2} B_{5}+A_{2}+A_{5}=0, \\
& R_{3443}=B_{3} B_{4}+A_{3}+A_{4}=0, \quad R_{3553}=B_{3} B_{5}+A_{3}+A_{5}=0 .
\end{aligned}
$$

从 (4.47), (4.48), 以及 (4.49) 的第一式可得

$$
\left(B_{4}-B_{5}\right) B_{1}=\frac{2 A_{124}^{2}}{\left(A_{1}-A_{2}\right)\left(A_{4}-A_{2}\right)} \neq 0, \quad B_{5} \neq 0 .
$$

所以在 $U$ 上, $B_{4} \neq B_{5}$. 结合 (4.49) 可知, 在 $U$ 上恒成立 $B_{3}=0$.

由于

$$
B_{1}+\cdots+B_{5}=0, \quad B_{1}^{2}+\cdots+B_{5}^{2}=\frac{4}{5}
$$


从 (4.48) 可以得到

$$
\begin{aligned}
& \left(B_{4}+B_{5}\right) B_{5}=-\left(B_{1}+B_{2}\right) B_{5}=\left(A_{1}+A_{2}+2 A_{5}\right), \\
& \left(\frac{4}{5}-B_{4}^{2}-B_{5}^{2}\right) B_{5}^{2}=\left(B_{1}^{2}+B_{2}^{2}\right) B_{5}^{2}=\left(A_{1}+A_{5}\right)^{2}+\left(A_{2}+A_{5}\right)^{2},
\end{aligned}
$$

或等价地

$$
\begin{aligned}
& B_{4} B_{5}=\left(A_{1}+A_{2}+2 A_{5}\right)-B_{5}^{2}, \\
& \frac{5}{4} B_{5}^{2}-\left(B_{4} B_{5}\right)^{2}-\left(B_{5}^{2}\right)^{2}=\left(A_{1}+A_{5}\right)^{2}+\left(A_{2}+A_{5}\right)^{2} .
\end{aligned}
$$

(4.51) 和 (4.52) 说明

$$
\begin{aligned}
2\left(B_{5}^{2}\right)^{2} & -2\left(\left(A_{1}+A_{2}+2 A_{5}\right)+\frac{5}{8}\right) B_{5}^{2} \\
& +\left(A_{1}+A_{2}+2 A_{5}\right)^{2}+\left(A_{1}+A_{5}\right)^{2}+\left(A_{2}+A_{5}\right)^{2}=0 .
\end{aligned}
$$

因此 $B_{5}$ 是常数. 由于 $B_{5} \neq 0$, 由 (4.48) 可知, $B_{1}$ 和 $B_{2}$ 也是常数. 因而由 (4.50), $B_{4}$ 是常数. 可见所 有的 Möbius 主曲率均是常数.

引理 4.12 如果在某个开集 $U \subset M^{*}$ 上, $A_{123} \neq 0$, 并且 $\left\{A_{124}, A_{125}, A_{134}, A_{135}, A_{234}, A_{235}\right\}$ 中 恰有三个元素不为零, 则 $x: U \rightarrow S^{6}$ 是 Möbius 等参超曲面.

证明 根据引理 4.5 , 只需考虑情形 $A_{123} A_{124} A_{134} A_{234} \neq 0$ 并且 $A_{125}=A_{135}=A_{235}=0$. 由 (4.9) 和引理 4.4 可知,

$$
\begin{aligned}
& \omega_{1}^{5}=\omega_{2}^{5}=\omega_{3}^{5}=0 \\
& R_{1551}=B_{1} B_{5}+A_{1}+A_{5}=0 \\
& R_{2552}=B_{2} B_{5}+A_{2}+A_{5}=0 \\
& R_{3553}=B_{3} B_{5}+A_{3}+A_{5}=0 .
\end{aligned}
$$

从 (4.53)-(4.56) 可得

$$
\begin{aligned}
& 0=d \omega_{1}^{5}-\omega_{1}^{k} \wedge \omega_{k}^{5}=-\omega_{1}^{4} \wedge \omega_{4}^{5}=-\left(\Gamma_{12}^{4} \omega^{2}+\Gamma_{13}^{4} \omega^{3}\right) \wedge \omega_{4}^{5}, \\
& 0=d \omega_{2}^{5}-\omega_{2}^{k} \wedge \omega_{k}^{5}=-\omega_{2}^{4} \wedge \omega_{4}^{5}=-\left(\Gamma_{21}^{4} \omega^{1}+\Gamma_{23}^{4} \omega^{3}\right) \wedge \omega_{4}^{5}, \\
& 0=d \omega_{3}^{5}-\omega_{3}^{k} \wedge \omega_{k}^{5}=-\omega_{3}^{4} \wedge \omega_{4}^{5}=-\left(\Gamma_{31}^{4} \omega^{1}+\Gamma_{32}^{4} \omega^{2}\right) \wedge \omega_{4}^{5} .
\end{aligned}
$$

因此, $w_{4}^{5}=0$. 此式结合 (4.53) 说明

$$
R_{4545}=B_{4} B_{5}+A_{4}+A_{5}=0 .
$$

从 (4.54)-(4.57) 可以得到

$$
B_{5}^{2}=-\left(B_{1}+B_{2}+B_{3}+B_{4}\right) B_{5}=A_{1}+A_{2}+A_{3}+A_{4}+4 A_{5}=\text { const. }
$$

显然, $B_{5} \neq 0$. 再由 (4.54)-(4.57) 可知, $B_{1}, B_{2}, B_{3}, B_{4}$ 是常数. 所以 $x: U \rightarrow S^{6}$ 是 Möbius 等参超曲 面.

命题 4.2 的证明 根据引理 4.8-4.12, 不难看出 $x$ 在 $M$ 的非空开集 $M^{*}$ 上是 Möbius 等参的. 再 结合 $M$ 的连通性可以说明 $M^{*}=M$, 因而 $x: M \rightarrow S^{6}$ 是 Möbius 等参超曲面. 


\section{5 定理 1.4 的证明}

设 $x: M \rightarrow S^{6}$ 是一个 Blaschke 等参超曲面.

如果 $x$ 具有不超过两个的不同 Blaschke 特征值, 则由定理 1.2, $x$ 局部地 Möbius 等价于定理 1.4 中所列超曲面 (1)-(8) 中的一个;

如果 $x$ 有超过两个的不同 Blaschke 特征值, 则由定理 1.1, 定理 1.3 和命题 4.2, $x$ 一定是 Möbius 等 参的. 从而由定理 4.1, $x$ 局部地 Möbius 等价于定理 4.1 中所列超曲面 (4), (5), (6)(也即定理 1.4 中所 列的超曲面 (9), (10), (11)) 中的一个.

作者感谢审稿人的有益评价和修改意见; 对于胡泽军教授就相关问题的有益讨论也深表谢意!

\section{参考文献}

1 Wang C P. Möbius geometry of submanifolds in $S^{n}$. Manuscripta Math, 1998, 96: 517-534

2 Liu H L, Wang C P, Zhao G S. Möbius isotropic submanifolds in $S^{n}$. Tohoku Math J, 2001, 53: 553-569

3 Li H Z, Wang C P. Möbius geometry of hypersurfaces with constant mean curvature and scalar curvature. Manuscripta Math, 2003, 112: 1-13

4 Li X X, Zhang F Y. A Möbius characterization of submanifolds in real space forms with parallel mean curvature and constant scalar curvature. Manuscripta Math, 2005, 117: 135-152

5 胡泽军, 李海中. $S^{n+1}$ 中具有平行 Möbius 第二基本形式超曲面的分类. 中国科学 A 辑, 2004, 34: 28-39

6 Li X X. Zhang F Y. A classification of immersed hypersurfaces in $S^{m+1}$ with parallel Blaschke tensors. Tohoku Math J, 2006, 58: 581-597

7 Cheng Q-M. Li X X. Qi X R. A classification of hypersurfaces with parallel para-Blaschke tensor in $S^{m+1}$. Int J Math, 2010, 3: $297-316$

8 钟定兴, 孙弘安. 单位球面上仿 Blaschke 张量的特征值为常数的超曲面. 数学学报, 2008, 51: 579-592

9 Li H Z, Liu H L, Wang C P, Zhao G S. Möbius isoparametric hypersurfaces in $S^{n+1}$ with two distinct principal curvatures. Acta Math Sin (Eng Ser), 2002, 18: 437-446

10 Li X X, Zhang F Y. On the Blaschke isoparametric hypersurfaces in the unit sphere. Acta Math Sin (Eng Ser), 2009, 25: $657-678$

11 Li X X, Zhang F Y. Immersed hypersurfaces in the unit sphere $S^{m+1}$ with constant Blaschke eigenvalues. Acta Math Sinica, Eng Ser, 2007, 23: 533-548

$12 \mathrm{Hu}$ Z J, Li H Z. A rigidity theorem for hypersurfaces with positive Möbius Ricci curvature in $S^{n+1}$. Tsukuba J Math, 2005, 29: 29-47

13 Hu Z J, Li H Z. Submanifolds with constant Möbius scalar curvature in $S^{n}$. Manuscripta Math, 2003, 111: 287-302

14 Li H Z, Wang C P. Surfaces with vanishing Möbius form in $S^{n}$. Acta Math Sin (Eng Ser), 2003, 19: 671-678

15 Li H Z, Wang C P, Wu F E. A Moebius characterization of Veronese surfaces in $S^{n}$. Math Ann, 2001, 319: 707-714

$16 \mathrm{Hu}$ Z J, Li D Y. Möbius isoparametric hypersurfaces with three distinct principal curvatures. Pacific Math J, 2007, 232: $289-311$

$17 \mathrm{Hu}$ Z J, Li H Z. Classification of Möbius isoparametric hypersurfaces in $S^{4}$. Nagoya Math J, 2005, 179: 147-162

$18 \mathrm{Hu} \mathrm{Z} \mathrm{J,} \mathrm{Li} \mathrm{H} \mathrm{Z,} \mathrm{Wang} \mathrm{C} \mathrm{P.} \mathrm{Classification} \mathrm{of} \mathrm{Möbius} \mathrm{isoparametric} \mathrm{hypersufaces} \mathrm{in} S^{5}$. Monatsh Math, 2007, 151: $202-222$

19 Hu Z J, Zhai S J. Classification of Möbius isoparametric hypersurfaces in the unit six-sphere. Tohoku Math J, 2008, 60: 499-526

20 Li X X, Peng Y J. Classification of Blaschke isoparametric hypersurfaces with three distinct Blaschke eigenvalues. Results Math, 2010, 58: 145-172, doi: 10.1007/s00025-010-0033-5

21 Blaschke W. Vorlesungen über Differentialgeometrie, vol. 3. Berlin: Springer, 1929

22 Cartan É. Sur des familles remarquables d'hypersurfaces isoparametriques dans les espace spheriques. Math Z, 1939, 45: 335-367

23 Cecil T, Chi Q S, Jensen G R. Isoparametric hypersurfaces with four principal curvatures. Ann of Math (2), 2007, 166: $1-76$

24 Cartan É. Familles de surfaces isoparamétriques dans les espace à courbure constante. Annali di Mat, 1938, 17: 177-191 


\section{The Blaschke isoparametric hypersurfaces in the unit sphere $S^{6}$}

LI XingXiao \& PENG YeJuan

Abstract An immersed umbilic-free submanifold in the unit sphere is called Blaschke isoparametric if its Möbius form vanishes identically and all of its Blaschke eigenvalues are constant. The classification of Blaschke isoparametric hypersurfaces of dimension $m \leqslant 4$ has already been done. Note that all known examples of Möbius isoparametric hypersurfaces are in fact Blaschke isoparametric. But there do exist many examples of Blaschke isoparametric hypersurfaces which are not Möbius isoparametric, and all those examples have no more than two Blaschke eigenvalues. In this paper we give a complete classification of Blaschke isoparametric hypersurfaces of dimension $m=5$, on the basis of known classification theorems for both the Blaschke and the Möbius isoparametric hypersurfaces. In particular, we shall prove that all the Blaschke isoparametric hypersurfaces in $S^{6}$ with more than two distinct Blaschke eigenvalues are necessarily Möbius isoparametric, providing another partial solution to the problem which asks whether or not a Blaschke isoparametric hypersurface with more than two Blaschke eigenvalues is necessarily Möbius isoparametric.

Keywords: Blaschke isoparametric hypersurface, Möbius form, Blaschke tensor, Möbius metric, Möbius second fundamental form

$\operatorname{MSC}(2000): \quad 53 \mathrm{A30}, 53 \mathrm{B25}$ 